\title{
High-frequency structure- and air-borne sound transmission for a tractor model using Dynamical Energy Analysis
}

\author{
Timo Hartmann ${ }^{\mathrm{a}}$, Satoshi Morita ${ }^{\mathrm{b}}$, Gregor Tanner ${ }^{\mathrm{a}, *}$, David J. Chappell ${ }^{\mathrm{c}}$ \\ ${ }^{a}$ School of Mathematical Sciences, University of Nottingham, Nottingham NG7 2RD, UK \\ ${ }^{b}$ Yanmar Co., Ltd., 2481, Umeghara, Maibara Shiga, 521-8511, Japan \\ ${ }^{c}$ School of Science and Technology, Nottingham Trent University, Nottingham NG11 \\ $8 N S, U K$
}

\begin{abstract}
Dynamical Energy Analysis (DEA) is a mesh-based high frequency method for modelling structure borne sound in complex built-up structures. Vibroacoustic simulations are performed directly on finite element meshes, circumventing the need for re-modelling strategies. DEA provides detailed spatial information about the vibrational energy distribution within a complex structure in the mid-to-high frequency range. We will present here progress in the development of the DEA method towards handling complex FE-meshes including Rigid Body Elements and sound radiation. We also provide, for the first time, a detailed comparison of the simulations with measurements on a complex engineering structure consisting of the chassis and cabin of a tractor. Both structure borne vibrations and sound pressure levels (SPL) inside the cabin were considered. For the latter, a combined DEA/SEA analysis has been developed. The simulation results compare favourably with measurement results, both for vibration levels measured across the structure and for SPLs inside the cabin.
\end{abstract}

Keywords: Vibro-acoustics, Statistical Energy Analysis, Ray Tracing, Transfer operators, high-frequency wave equations

\footnotetext{
* Corresponding author

Email address: gregor.tanner@nottingham.ac.uk (Gregor Tanner)
} 


\section{Introduction}

A major difficulty in modelling structure-borne sound lies in the complex geometry of the structures. The Finite Element Method (FEM) can describe geometric details with sufficient accuracy in the low frequency region, but requires extremely fine meshes at high frequencies to capture the shorter wavelengths. Statistical representations such as the Statistical Energy Analysis (SEA) [1] have been developed, leading to relatively small and simple models in comparison with FEM. A range of methods have been proposed to extend the applicability of SEA such as the hybrid FEM/SEA method $[2,3,4]$. An alternative to SEA is to start from a ray-tracing ansatz and reformulate it in terms of integral equations. This leads to linear flow equations for the mean vibrational energy density and forms the basis of the Dynamical Energy Analysis (DEA) method introduced in [5]. DEA includes SEA as special case via a low order representation of the so-called transfer operator. Higher order implementations enrich the DEA model with information from the underlying ray dynamics, leading to a relaxation of SEA assumptions. In particular, DEA allows for more freedom in sub-structuring the total system and variations of the energy density across sub-structures can be modelled. An efficient implementation of DEA on meshes has been presented in $[6,7]$. Vibro-acoustic energy densities are computed for multi-modal propagation, including energy transport over curved surfaces. Connections at material interfaces are described in terms of reflection/transmission matrices and hence DEA resolves the full geometrical complexity of the structure. DEA implemented on meshes provides a detailed spatial resolution of the energy density, including variations over larger substructures such as the windows due to damping or local coupling.

In this paper, we apply the DEA method to a stripped down version of a Yanmar tractor consisting of the cabin (including windows and doors) mounted on a chassis. The simulations are carried out in the range from 400 to $4000 \mathrm{~Hz}$ both for the structure borne sound as well as the sound pressure level (SPL) at the driver's ear position. The results are compared with detailed measurements across the entire structure. The DEA results are based on an FE mesh created for the structure with standard meshing software. In this paper, the main focus is on the implementation of DEA in the presence of Rigid Body Elements (RBEs), or similar FE coupling methods [8]. We will furthermore introduce a DEA/SEA hybrid method for determining the acoustical response inside the cabin. In Sect. 2, we introduce the built-up structure and give a detailed account of the measurement program. In Sect. 3, we describe the DEA technique and discuss our strategies for dealing with 


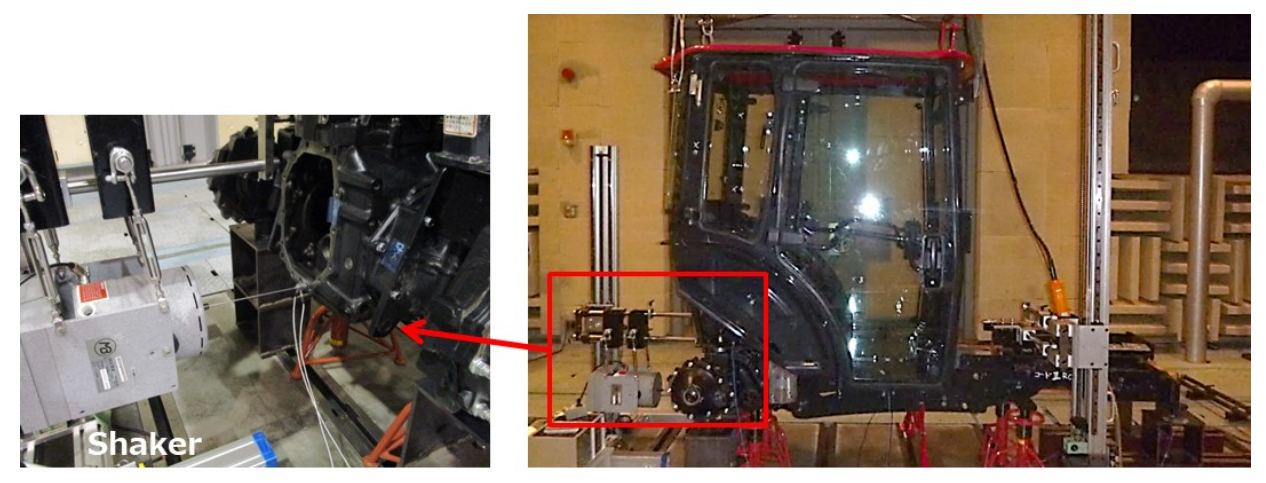

Figure 1: Body-in-Blue structure together with the excitation region

mesh interconnections, such as the RBEs commonly used in an FE analysis to join together different sub-meshes of the structure. We also detail the interior acoustic predictions via the DEA/SEA hybrid method mentioned above. Results will be presented and discussed in Sect. 4. A detailed account on implementing DEA on 2D meshes is given here for the first time in Appendix A to Appendix D.

\section{The tractor - measurement set-up}

The tractor model under consideration in this study has been provided by Yanmar Co., Ltd. and is a stripped down version of a tractor from Yanmar's EG400 series. The tractor body consists of a chassis frame and a cabin; the latter includes doors and windows, often referred to as a 'body-in-blue' (BiB) structure. The chassis frame consists of the gear casing and a front frame. The cabin is mounted onto the chassis by four rubber mounts; the actual structure is depicted in Fig. 1, together with the excitation point (to the rear) provided by a modal shaker in the frequency range between $400 \mathrm{~Hz}$ to $4000 \mathrm{~Hz}$. The chassis frame is supported by rubber mounts to ensure a free-free boundary condition.

The sound pressure at the operator's ear location is measured with a microphone. In addition, the acceleration of the structure is measured at 29 points on the cabin and at 13 points on the chassis frame using a accelerometers. The acceleration on the upper and lower sides of each cabin mount are also measured giving valuable information about the coupling via the rubber mounts. Fig. 2 shows the location of the measurement points (blue dots) on an FE model of the structure. The FE mesh, also used for the 


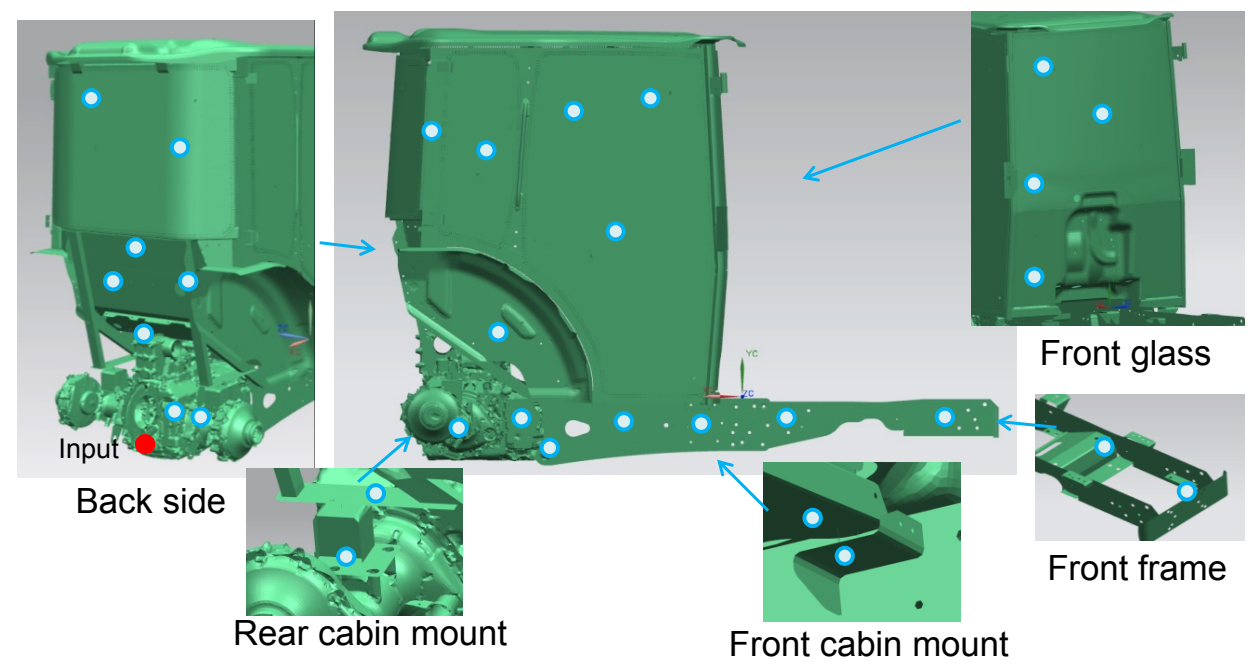

Figure 2: FEM model of the tractor including the accelerometer positions across the structure (blue dots, 44 points in total).

DEA computation in Sect. 4, consists of 521572 mesh cells with a typical cell measuring $1 \mathrm{~cm}$ in diameter. In the FE model, the rubber mounts are treated as spring elements, so-called CELAS1 elements [8].

\section{Dynamical Energy Analysis on meshes}

\subsection{General theory}

We will give here a brief account of the major ideas behind DEA - for a detailed description of the theory and the implementation on $2 \mathrm{D}$ meshes, see [6] and Appendix A to Appendix C. In the high frequency regime, the dynamics described by linear wave equations can be approximated using semi-classical or ray-tracing methods describing the transition from wave acoustics to ray acoustics; this serves as a starting point for DEA. By neglecting phase information, DEA approximates the transport of wave energy by a Hamiltionian flow [5] described by the Liouville equation. DEA, like SEA, can thus determine averaged values of the local wave energy neglecting variations on a wavelength scale. The energy flow is phase space volume preserving and can be formulated in terms of trajectories or rays. In contrast to ray tracing, DEA does not work with individual rays, but describes how ray densities are transported along the flow. In this work we are primarily 
interested in the stationary solution of transport problems corresponding to wave problems in the frequency domain with time-harmonic driving terms. Interference (or phase) effects are neglected.

The ray density can be related to a wave energy density and is defined on a phase space, that is, it depends both on position and direction. For two-dimensional systems (as considered in this work), the phase space is four dimensional and is parametrised by two position and two momentum coordinates. The momentum coordinates are equivalent to the wave vector. For fixed frequency $\omega$ and a given wave mode, the modulus of the wave vector, that is, the wavenumber, is fixed. Hence, there is only one free parameter necessary to describe the direction.

The transport problem can be conveniently solved on a mesh as described in [6]. The meshes can be quite large such as the mesh shown in Fig. 2 with more than 250000 mesh points and are typically reused from existing FE meshes. There is thus no need for sub-structuring and typical structural elements and interfaces will be resolved in detail by the mesh. It is advantageous to restrict the Hamiltonian flow to sections on the union of all the edges of the mesh cells describing the two-dimensional structure in question. The coordinates used to describe this restricted two-dimensional phase space, the so-called Surface of Section (SoS), are the arc-length along each edge $i, s_{i}$, and the corresponding component of the wave vector along the direction of the edge, $p_{i}$. The continuous Hamiltonian flow is thus transformed into a discrete map, also called the Discrete Flow Map (DFM) [6].

DEA describes the transport of ray densities from the SoS to itself using a linear transfer operator. Iterations of this operator map phase space densities on the SoS back to itself; the stationary solution is obtained by summing over all iterations of an initial density produced by a source term, see Appendix A for details. To relate the source density to an SoS boundary density and to obtain the stationary density in the interior of the mesh cells, phase space densities on the SoS need to be related back to the corresponding full four-dimensional phase space density; this step is described in Appendix B. The evaluation of densities in the interior and their relation to the boundary densities is treated in Appendix C.

Mode conversion between in-plane and flexural waves at boundaries can be included in the treatment and the reflection/transmission coefficients are obtained from wave scattering theory [9]. An example is shown in Fig. A.14 in Appendix A. Shell effects leading to curved rays [10] are included by treating the meshed structure as a set of plate-like elements, see $[6,7]$ for details. Modelling connections between different meshes via RBEs, such as used in the tractor model, is described briefly in the next section and in 


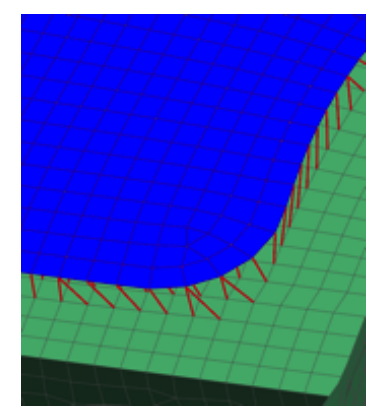

Figure 3: A typical RBE-interface connecting two meshes together.

more detail in Appendix D.

\subsection{Implementation of RBEs in DEA}

DEA, as described in Sect. 3.1 above and in more detail in Appendix A, models the flow of wave energy across a triangulated surface, and in particular, the energy flow between neighbouring mesh cells through their common edges. FE meshes used in engineering often consist of several triangulated surfaces coupled together through so-called Rigid Body Elements (RBEs), see Fig. 3. The RBEs correspond to linear constraints between the displacements at several points on the mesh [8], and as a result, energy can flow between locations on the mesh that have no physical connection (such as a common edge). These elements cannot be used in an ordinary DEA treatment, which is based purely on energy flux through interfaces. The FE model of the tractor structure shown in Fig. 2 is such an example, consisting of a number of different sub-meshes connected via RBEs. The number of RBEs can be quite large, 7481 RBEs in the case of the FE mesh in Fig. 2. The RBEs are used here to form connections at the glass-metal interfaces, the sidewall-roof interfaces, and for connecting the doors to the cabin frame.

To avoid an expensive re-meshing for DEA, we have developed a method treating RBEs as DEA coupling elements directly, so-called RBE patches; these patches can handle energy transfer through RBEs and similar constraint elements. In ordinary DEA, where energy flows between neighbouring mesh cells, one can make a local plane wave assumption. That means when a plane wave with a well-defined direction hits an edge, it comes out on the other side with a well-defined direction. This scenario directly translates to the ray picture. In actual FE meshes, the RBEs often connect different points in a very complicated way and we have, in general, little or no in- 


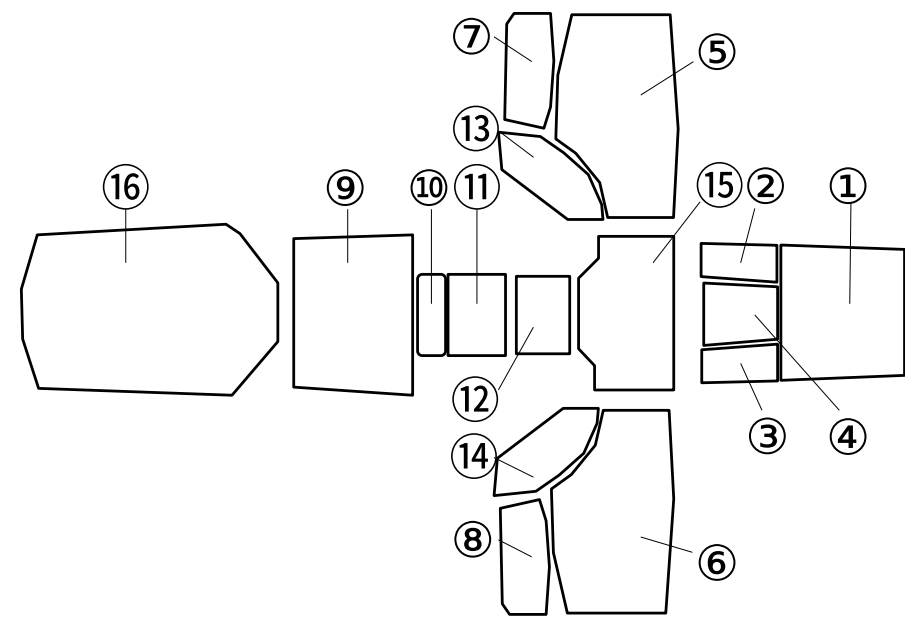

Figure 4: SEA subsystems of the tractor cabin

formation about how rays encountering an RBE will emerge on the other side. Due to this lack of information, we make the simplifying assumption that an RBE patch in DEA spreads out the incoming energy uniformly in all directions on both meshes connected by the RBE. In the ray picture, that means that a single ray with a well-defined direction is split into many rays going into many directions on both ends of the RBE, keeping the total (energy) density preserved. Details of the mathematical implementation of RBE patches are given in Appendix D.

To describe the energy transfer across non-compatible meshes in our tractor model we use RBE patch elements at each RBE position, thus introducing coupling between edges both in the 'upper' and 'lower' sheets connected by the RBE. Incoming ray-densities on one side of the RBE-interface are then mapped onto the other side of the interface. In the FE model, the rubber mounts between the chassis and the cabin are treated as CELAS1 spring elements [8]. For the purpose of DEA, we treat these elements in the same way as we treat RBEs.

\subsection{Sound pressure levels in the interior of the cabin - a hybrid SEA/DEA approach}

The sound pressure level (SPL) inside the cabin, and in particular at the position of the driver's ear, can be computed from the vibrational energy densities obtained in the DEA computation. Ideally, this would be done 
by coupling the $2 \mathrm{D}$ structural DEA computation to a $3 \mathrm{D}$ acoustics DEA model, such as described in [14]. However, a fluid-structure model has yet to be fully developed within DEA and further simplifying assumptions are necessary at this point. We present an SEA/DEA hybrid method in this section, which computes the SPLs inside the cabin using an SEA model where the radiated energy produced by the bending vibrations of the cabin walls, floor and ceiling obtained from DEA provide the input/source. The SEA treatment inherently implies a diffuse field assumption, that is, the steady-state interior sound field (including wall reflections) is assumed nondirectional.

To obtain the SEA sub-systems, we first identify the main panels forming the walls of the cabin as displayed in Fig. 4. We then define a power flow equation between these panels (the subsystems) and the acoustic volume of the cabin (the cavity). Only the coupling between the cavity and each main panel is taken into account; the coupling between different panels is already treated through calculating the vibrational energy distribution on the panels using DEA. The resulting power flow equations can then be written as

$$
\left(\begin{array}{c}
P_{1} \\
P_{2} \\
\vdots \\
P_{n} \\
P_{c}
\end{array}\right)=\omega\left[\begin{array}{ccccc}
\eta_{c 1} & 0 & \cdots & 0 & -\eta_{1 c} \\
0 & \eta_{c 2} & \cdots & 0 & -\eta_{2 c} \\
\vdots & \vdots & \ddots & \vdots & \vdots \\
0 & 0 & \cdots & \eta_{c n} & -\eta_{n c} \\
-\eta_{c 1} & -\eta_{c 2} & \cdots & -\eta_{c n} & \eta_{c}^{d}+\sum_{i} \eta_{c i}
\end{array}\right]\left(\begin{array}{c}
E_{1} \\
E_{2} \\
\vdots \\
E_{n} \\
E_{c}
\end{array}\right)
$$

Here, $\eta_{c}^{d}$ is the damping loss factor in air, that is, inside the cavity and $\eta_{i c}, i=1,2, \ldots, n$, are the coupling loss factors between the structural subsystem $i$ and the cavity subsystem $c$ such that $\eta_{i c}=\eta_{c i}$. In addition, $E_{i}$ is the vibrational energy in panel $i, P_{i}$ is an external input power flowing into panel $i$ from other parts of the structure, and $E_{c}$ is the acoustic energy in the cabin. Under the assumption of stationarity of the vibrational field in the structure, we can set

$$
P_{i}=\omega \eta_{c i} E_{i}^{0}=\omega \eta_{c i} M_{i}<v_{i}^{2}>
$$

where $E_{i}^{0}$ is the energy in panel $i$ without acoustic coupling. The coupling loss factors are given by the radiation condition

$$
\eta_{c i}=\frac{Z_{0} A_{i} \sigma_{i}^{r a d}}{\omega M_{i}}
$$

Here, $Z_{0}$ is the acoustic impedance, $A_{i}$ is the area of the $i$ th plate and $\sigma_{i}^{r a d}$ is the corresponding radiation efficiency for a rectangular plate, which is 


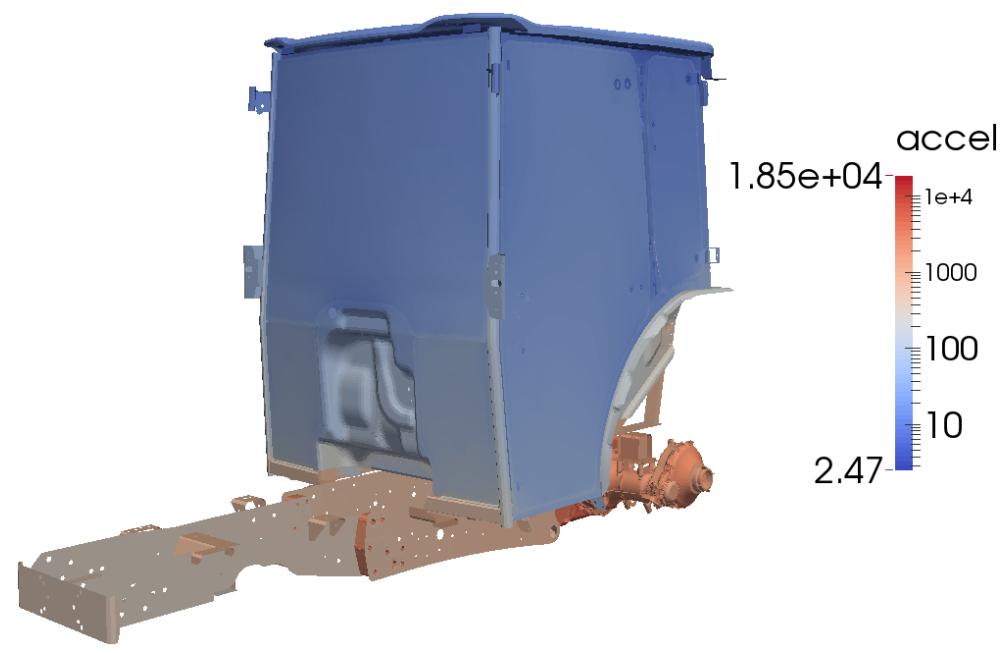

Figure 5: DEA results for the acceleration (given here in $\mathrm{mm} / \mathrm{s}^{2}$ ) at a frequency of 1000 $\mathrm{Hz}$.

computed using the approximation derived in [11]. The mean displacement velocity $\left\langle v_{i}^{2}\right\rangle$ in panel $i$ with mass $M_{i}$ is obtained from the DEA calculation of the flexural mode and averaged over each of the panels. After setting $P_{c}=0$, we solve Eq. (1) for the energies $E_{i}, i=1,2, \ldots, n$, and $E_{c}$. For the form of the SEA matrix given in Eq. (1), the result can easily be given in closed form, that is,

$$
E_{c}=\frac{1}{\omega \eta_{c}^{d}} \sum_{i=1}^{n} P_{i} .
$$

The acoustic energy inside the cabin is the sum of the inflowing energies from all sub-systems weighted by the inverse of the damping coefficient. The latter can be interpreted as a trapping coefficient taking care of reverberation inside the cabin. The mean SPL inside the cabin is then obtained from the value of $E_{c}$. For the example considered here, we divide the cabin walls, ceiling and floor into 16 sub-panels as shown in Fig. 4. We assume furthermore that the damping loss in air is $\eta_{c}^{d}=1.5 \%$; damping within the structure is already included in the DEA computation as discussed in Sec. 4.2 . 


\section{Results}

\subsection{Structure-borne sound calculations}

We perform DEA calculations for the full structure shown in Fig. 2 in the frequency range $400 \mathrm{~Hz}-4000 \mathrm{~Hz}$. The damping values and input powers used in the numerical simulation are described in the next section. The results are compared to experimental data obtained from measurements carried out by Yanmar. The experimental results are averaged over one third octave bands and the corresponding DEA results are calculated at the centre frequencies of these bands (without averaging). The DEA computation is preformed on a mesh consisting of 521572 elements and for a momentum basis of Legendre polynomials truncated at degree 3; typical computation times are about $12 \mathrm{~h}$ for a single frequency on a single core of a desktop PC. Note that the resolution for a full frequency run as shown, for example, in Fig. 7 does not need to be high and can be done with a few dozen points, here 10 frequencies in the range from $400 \mathrm{~Hz}$ to $4000 \mathrm{~Hz}$.

Fig. 5 shows the outcome of a DEA calculation at $1000 \mathrm{~Hz}$. DEA computes vibrational energy densities for different wave modes as laid out in Appendix C; these energy densities can be related to velocities for flexural displacements and thus to acceleration levels resulting from a narrow-band excitation of the structure, which can then be compared directly with the measurement results. Fig. 5 shows the local variations which can be obtained by implementing DEA on an FE mesh. Note that no additional work regarding sub-structuring is necessary; DEA serves as a black-box tool, once meshes have been read in and interpreted correctly (such as with respect to RBE coupling) - this can all be automised and requires no further user input. A more in-depth analysis of Fig. 5 shows that most of the vibrational energy remains near the source (at the rear end of the chassis) and in the chassis itself. This is due to the weak coupling between the chassis and the cabin, facilitated by the rubber mounts. The acceleration levels on the cabin are thus considerably lower overall and decrease according to the distance from the source.

A point-by-point comparison with the measurement results at $2500 \mathrm{~Hz}$ can be found in Fig. 6. We note that the numerical results agree reasonably well with the measurements across the whole structure and display a level of detail not easily achievable with other high-frequency methods. There are systematic differences, for example at the rubber mounts and on the frame elements; these may be explained by the complex nature of the mounting points and the beam-like structure of the frame, both of which require further investigation. Results for specific measurement positions across the 


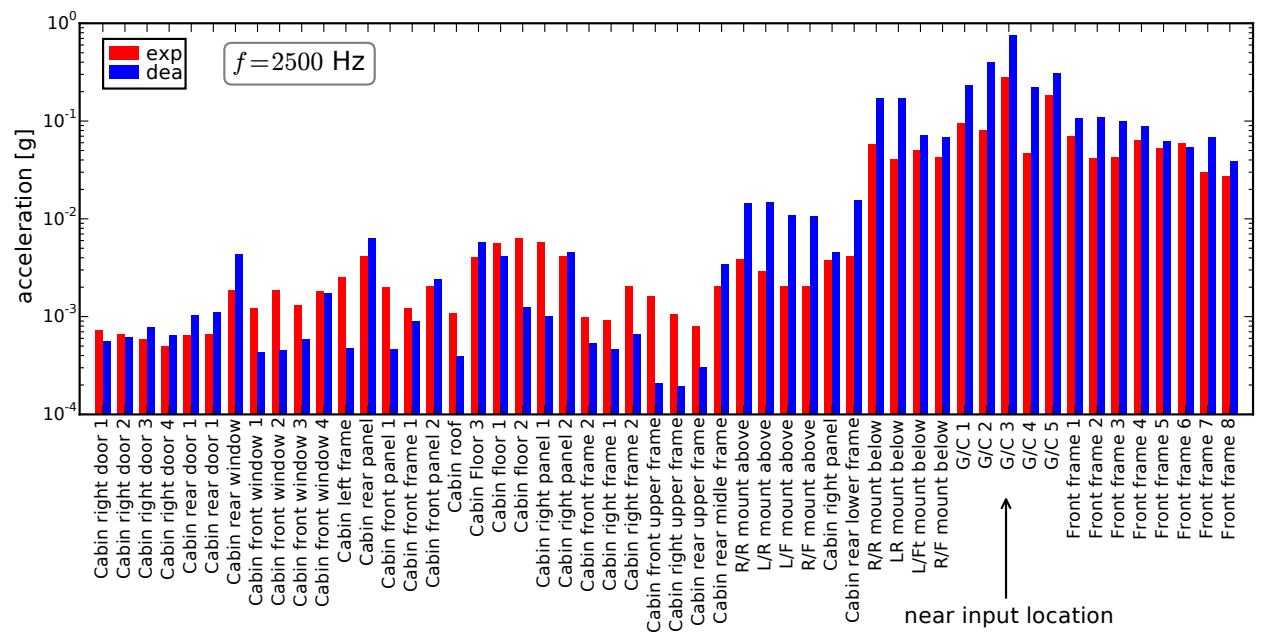

Figure 6: Comparison of simulation and measurement results at $2500 \mathrm{~Hz}$. The acceleration is measured in $\mathrm{g}=9.8 \mathrm{~m} / \mathrm{s}^{2}$.

whole frequency range are shown in Fig. 7. Good agreement is achieved and the energy gap between the chassis and the cabin is well captured. Overall, the simulation reproduces the energy distribution across the whole structure remarkably well despite the simplifying assumptions for the RBE coupling and the cabin mounts.

\subsection{Damping values and input power}

In addition to material parameters such as the Young's modulus, Poisson ratio, material densities and plate thickness, the material damping parameters play a crucial role in DEA calculations. We can distinguish two different types of damping mechanism here: firstly, the damping of vibrational energy as waves propagate across the structure (or across the mesh cells in our model), which is modelled by a hysteretic damping parameter. Secondly, damping occurs at rubber elements such as the rubber mounts supporting the cabin or the rubber seals connecting the metal frame with the window glass panes. These connections are treated with RBE patch elements and interface specific damping parameters are assigned.

Below $1600 \mathrm{~Hz}$, we are using a hysteretic damping value of 0.005 typical for metallic plates. Above $1600 \mathrm{~Hz}$, however, a hysteretic damping value of $0.5 \%$ has proven to be too large and associated DEA calculations underestimate the measurement results considerably. To obtain realistic high frequency damping parameters, we use the decrease in the measured accel- 


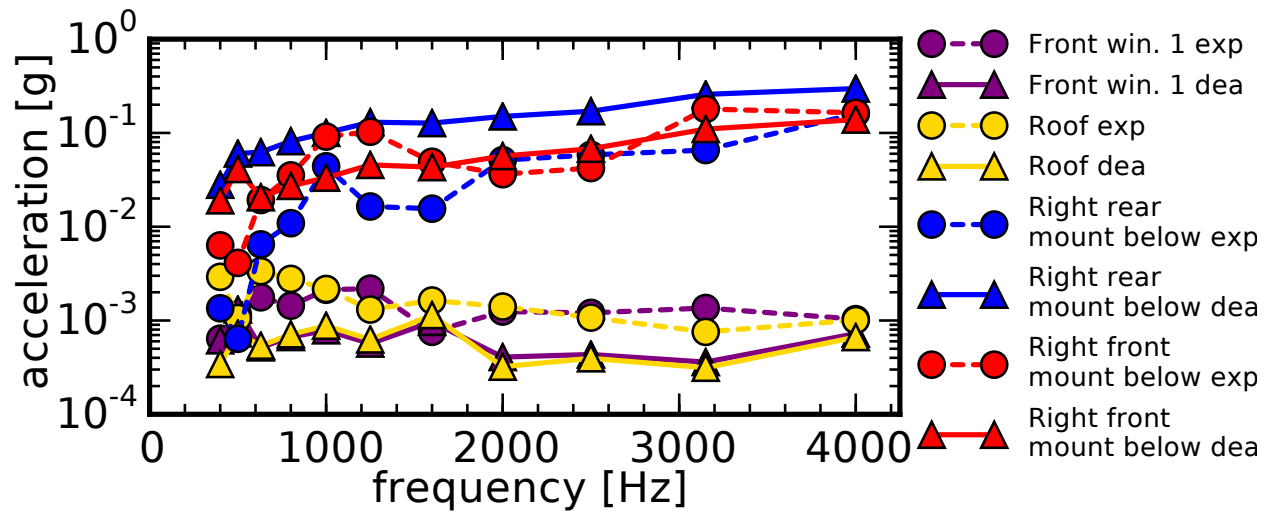

Figure 7: Acceleration at specific points on the structure: DEA results versus measurement over the frequency range $400 \mathrm{~Hz}$ to $4000 \mathrm{~Hz}$.

eration along the chassis, from the back to the front, to estimate frequency dependent hysteretic damping values. From this, we conclude that the hysteretic damping values need to be reduced from $0.5 \%$ at $1600 \mathrm{~Hz}$ to $0.1 \%$ at $4000 \mathrm{~Hz}$, see Fig. 8.

In the FE model of the tractor, the RBEs are always applied in places where the physical model has some material with damping properties. For example, between the windows and the cabin frame there is a sealing material. Therefore, we should assign an inherent damping parameter to the corresponding RBE, which is then used in Eq. (D.3). Currently we work with a transmission factor of 0.98 (across all frequencies) for all RBEs not associated with the rubber mounts. This value gives a reasonable agreement between measurement and DEA across all frequencies and points. We note that a transmission factor of 0.98 is fairly high and can not be related to a physical transmission factor. Instead, it reflects some of the properties of our RBE coupling implementation as described in Appendix D. The RBE interfaces are quite irregular as shown, for example, in Fig. 3. An important consequence of this irregularity is that energy can become trapped in the region near the RBEs for a relatively long time, which artificially increases the damping associated with the RBEs. Therefore the physically relevant damping across the window seals is larger than a transmission factor of 0.98 would suggest.

The RBEs describing the rubber mounts are treated differently and again we use the experimental data for realistic estimates of the damping behaviour. The rubber mounts are critical in any computation, as they are 

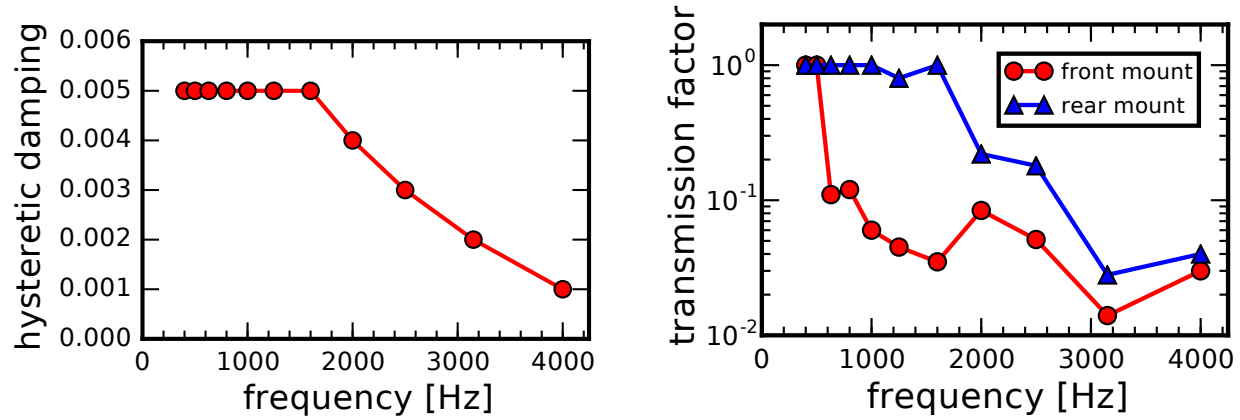

Figure 8: Left: Hysteretic damping values used in the DEA calculation. Right: Transmission factors for the rubber mounts used in DEA calculation.

designed to block structure-borne sound from flowing out of the chassis and into the cabin. In fact, they are the only places where such a vibrational energy transfer can take place. We denote the vibrational wave energy below and above the rubber mounts as $E_{<}$and $E_{>}$, respectively. We now consider the ratio $\tau=E_{>} / E_{<}$, which can be obtained directly from the measurement data and define the transmission factor in Eq. (D.3) as $\min (2 \tau, 1)$. Here, we have assumed an approximate linear relationship between the transmission factor and the energy ratio, and the factor of two is a consequence of the RBE patch element in DEA taking into account both reflected and transmitted energy. The results for $\tau$ are shown in Fig. 8. The transmission factors at the rear and front mounts are different, but coincide for the left and right mounts. The transmission factor reflects the steep decrease in acceleration measured from the lower (chassis) to the upper part (cabin) of the structure near the rubber mounts. As shown in Fig. 8, the damping properties of the rubber mounts vary greatly with frequency and with the applied load (as can be seen in the difference between the rear and front mounts). The damping parameters have entered the computation leading to the results presented in Figs. 6 and 7. The uncertainty in assigning the correct damping characteristics is shared with other numerical methods such as the FEM and hence the procedure for obtaining realistic damping parameters using measurements described here is not peculiar to DEA. The DEA method itself has no modelling capability to predict damping values and must rely on input from other pre-processing tools.

The vibrational energy determined by DEA scales linearly with the input power $P_{i n}$. In the experiment, the shaker at the rear of the gearbox excites the structure and thus provides a power input. We calculate 


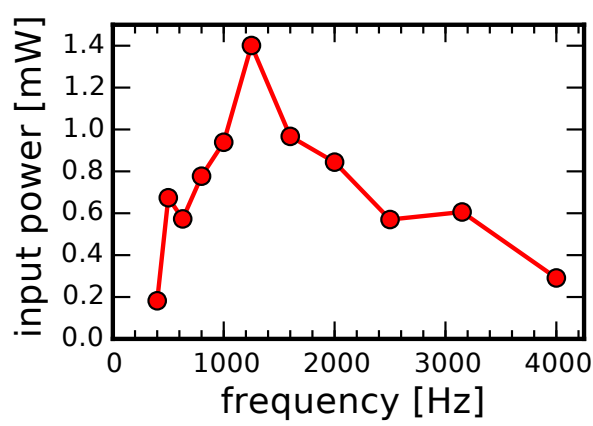

Figure 9: The measured input power $P_{i n}$ flowing from the shaker to the tractor.

$P_{\text {in }}=\frac{1}{2} \operatorname{Re}\left(\mathbf{v}^{*} \cdot \mathbf{F}\right)$ from the measured velocity $\mathbf{v}$ and force $\mathbf{F}$ at the point on the structure where the shaker is attached. The results are shown in Fig. 9. In the DEA calculation we apply a point source according to Eq. (C.9) at the appropriate mesh point, where the value of the input power $P_{i n}$ is determined from the measurements.

\subsection{Vibrational energy flow in the tractor - main transfer paths}

DEA calculates a ray density $\varrho(\mathbf{r}, \mathbf{p})$ in phase space consisting of a position variable $\mathbf{r}$ and a direction variable $\mathbf{p}$, see Appendix A to Appendix $\mathrm{C}$ for details. The structure under consideration here is effectively made up of two-dimensional plates and hence both $\mathbf{r}$ and $\mathbf{p}$ are two-dimensional, albeit on the surface of a complex structure in three dimensions. The results shown in Sect. 4.1 are all derived from energy density calculations, where the energy density $\epsilon(\mathbf{r})$ is proportional to the density of rays passing through the point $\mathbf{r}$, independent of the direction, that is,

$$
\epsilon(\mathbf{r}) \propto \int \varrho(\mathbf{r}, \mathbf{p}) \mathrm{d} \mathbf{p}
$$

see also Eq. (C.1). Note that for elastic waves we have different wave modes, that is, longitudinal, shear and bending modes and associated energy densities. While the DEA computation determines the contributions of all three modes, we consider here only the bending component since this gives the main contribution when compared to measured accelerations and interior SPLs. Furthermore, for a monochromatic excitation, the modulus of the momentum coordinate $|\mathbf{p}|=k(\omega)$ (that is, the wavenumber) is fixed for a fixed frequency $\omega$, but differs depending on the mode type. 

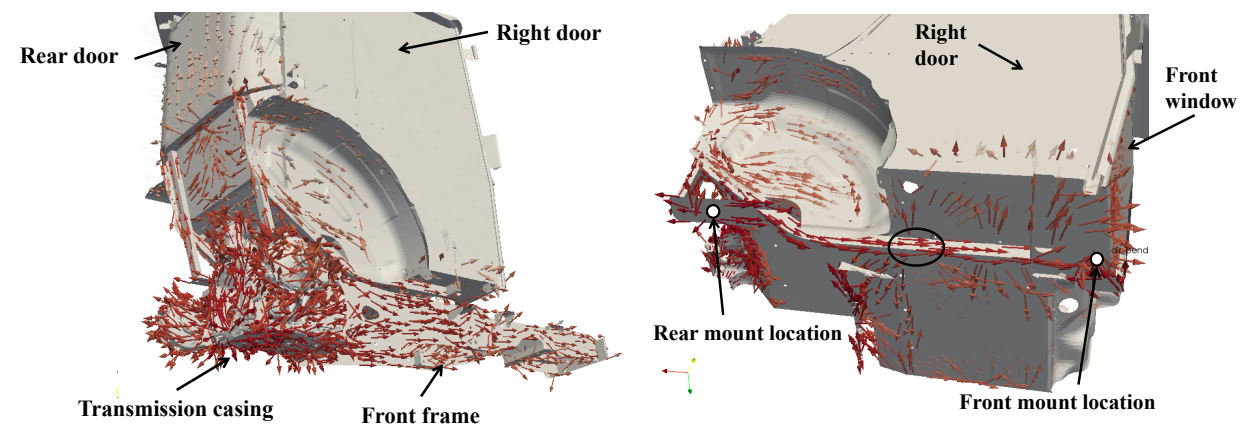

Figure 10: Left: Vibrational energy flow on the structure at $2500 \mathrm{~Hz}$; Right: Vibrational energy flow on the cabin main frame (Black circle: connection between main frame and floor panel).

The ray density $\varrho(\mathbf{r}, \mathbf{p})$ computed in DEA provides more information than just the energy density $\epsilon$, however. In particular, it allows one to estimate the momentum density vector $\mathbf{I}(\mathbf{r})$ directly, and thus give information about the direction of mean energy flow at each point on the structure. This can be done as a post-processing step by computing the vectorial quantity

$$
\mathbf{I}(\mathbf{r}) \propto \int \mathbf{p} \varrho(\mathbf{r}, \mathbf{p}) \mathrm{d} \mathbf{p}
$$

at each point on the structure; see also Eq. (C.5) and the discussion in Appendix C.2. This may serve as a valuable tool for the engineer and practitioner to identify important transfer paths, which can be used to inform optimisation measures. Results for the energy flow density on the tractor are shown in Fig. 10. On the left hand side of Fig. 10 we can see that the energy is spreading predominantly from the source region at the rear of the structure towards the front of the chassis. There is also directed energy spreading on the cabin, as shown on the right hand side of Fig. 10. In particular, one can detect energy flowing along the beam connecting the wheel area to the front of the cabin from which considerable amount of energy is spread into the front floor region. This information can be helpful for informing the location of sound insulation measures.

\subsection{SPL at driver's ear}

SPL calculations at the driver's ear position follow the approach sketched in Sect. 3.3. Results are presented in Fig. 11 and are compared with measurements. The hybrid SEA/DEA simulations and the measurements show 


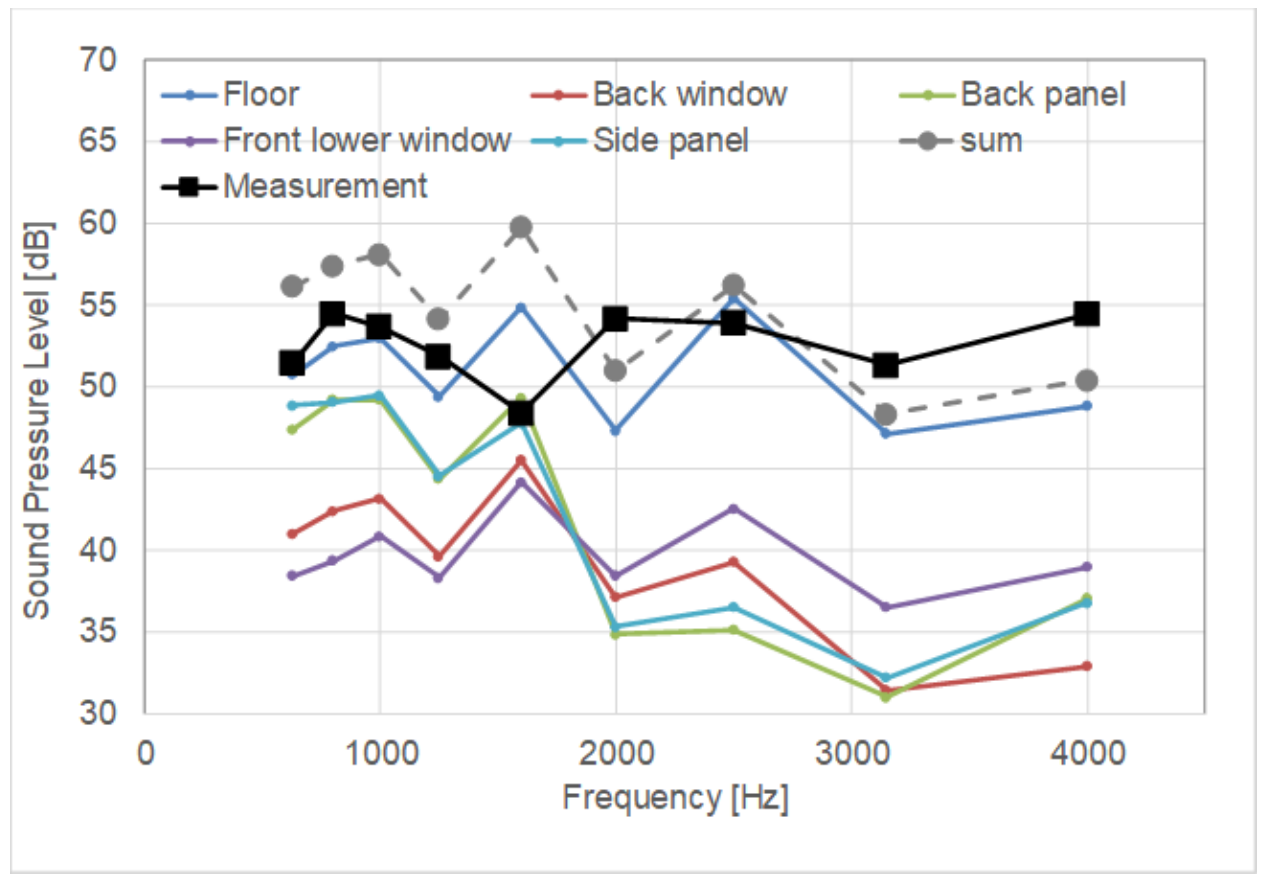

Figure 11: SPL at the driver's ear position: experiment (black, solid); SEA/DEA simulations (grey, dashed). The contribution from some selected panels are shown in colour; the main contributions are coming from the floor panel.

good agreement on average across the whole frequency range. We note variations in both the experimental data and the simulations, but the overall range (between 45 and $55 \mathrm{~dB}$ ) is captured well by the simulation. We also show individual contributions from selected plates; these contributions are computed by solving the SEA equations using a source term derived from the vibration levels of a given selected plate only. By far the most important contributions to the SPLs at the driver's ear come from the floor panel, that is, the panel closest to the source. Other important contributions are from the front and back windows and the side panels.

The agreement between measurements and calculations for the SPLs is slightly inferior compared to the vibration levels of the structure as for example shown in Fig. 6. This is nor surprising as additional approximations have been made here. For example, the radiation efficiencies are in itself an approximation and furthermore are originally defined only for rectangular, flat plates; we are using it here for sub-structures which are not necessarily completely flat or rectangular. 


\section{Conclusions}

We have demonstrated that the DEA method can compute structureborne sound across a complex structure, here for the case of a BiB substructure of a full tractor. The results presented in this work emphasise the level of detail provided by the DEA method and its flexibility in handling FE meshes including RBEs. Results over the full frequency range from $400 \mathrm{~Hz}$ to $4 \mathrm{kHz}$ have been presented and a new energy flow chart technique has been introduced which allows to obtain information about transfer paths in a post-processing step. A combined SEA/DEA model of the SPL in the interior of the cabin gives agreement within $\pm 5 \mathrm{~dB}$.

\section{Acknowledgements}

We thank Dimitrios Chronopoulos for helpful and interesting discussions. Support from the EU (FP7 IAPP grant no. 612237 (MHiVec)) is gratefully acknowledged. Financial support by Yanmar Co., Ltd. is thankfully acknowledged by TH and GT.

[1] R. H. Lyon, and R. G. DeJong, Theory and Application of Statistical Energy Analysis, Butterworth-Heinemann, Boston (1995).

[2] P. J. Shorter and R. S. Langley, Vibro-acoustic analysis of complex systems, J. Sound Vib., 288, 669-699, 2005.

[3] V. Cotoni, P.J. Shorter, and R.S. Langley, Numerical and experimental validation of a hybrid finite element-statistical energy analysis method, J. Acoust. Soc. Am., 122, 259-270, 2007.

[4] G. Xie, L. Dunne, A. Secgin and A. Zoghaib, Mid-frequency modelling of a car floor structure with hybrid method and SEA, International Symposium on the Computational Modelling and Analysis of Vehicle Body Noise and Vibration, 2012, Sussex, UK.

[5] G. Tanner, Dynamical energy analysis - Determining wave energy distributions in vibro-acoustical structures in the high-frequency regime, J. Sound Vib. 320, 1023-1038, 2009.

[6] D. J. Chappell, G. Tanner, D. Löchel and N. Søndergaard, Discrete flow mapping: transport of phase space densities on triangulated surfaces, Proc. R. Soc. A, 469, 20130153, 2013. 
[7] D. J. Chappell, D. Löchel, N. Søndergaard and G. Tanner, Dynamical energy analysis on mesh grids: A new tool for describing the vibro-acoustic response of complex mechanical structures, Wave Motion 51, 589-597, 2014 .

[8] NX Nastran User's Guide, https://docs.plm. automation. siemens. com/tdoc/nxnastran/10/help/\#uid:index.

[9] R. S. Langley and K. H. Heron, Elastic wave transmission through plate/beam junctions, J. Sound Vib. 143, 241, 1990.

[10] A. N. Norris and D. A. Rebinsky, Membrane and flexural waves on thin shells, ASME J. Vib. Acoust. 116, 457-467, 1994.

[11] G. Maidanik, Response of ribbed panels to reverberant acoustic fields, J. Acoust. Soc. Am. 34, 809-826, 1962.

[12] J. Bajars, D. J. Chappell, T. Hartmann, G. Tanner, Improved approximation of phase-space densities on triangulated domains using Discrete Flow Mapping with p-refinement, J. Sci. Comp. 72, 1290-1312, 2017.

[13] D. J. Chappell and G. Tanner, Solving the stationary Liouville equation via a boundary element method, J. Comp. Phys., 234, 487-498, 2013.

[14] J. Bajars, D.J. Chappell, N. Søndergaard and G. Tanner, Transport of phase space densities through tetrahedral meshes using discrete flow mapping, Journal of Computational Physics 328, 95-108, 2017.

\section{Appendix A. DEA implementation}

We present here a detailed account of the implementation of DEA on meshes as sketched in Sect. 3.1. In this work we only consider meshes consisting of two dimensional elements (eventually glued together with RBEs) and polygonal mesh cells. Energy can flow between neighbouring mesh cells, which are joined along edges, and we describe the densities of rays on the edges of each mesh cell. In order to parametrise rays starting at an edge and travelling in any direction to one of the other edges, each edge is split into several half-edges. Each half-edge belongs to exactly one of the polygons joined together along the edge and covers all rays originating at the edge and going into that polygon. That means each half-edge covers $180^{\circ}$. For edges connecting two mesh cells, the two half-edges together cover $360^{\circ}$. 


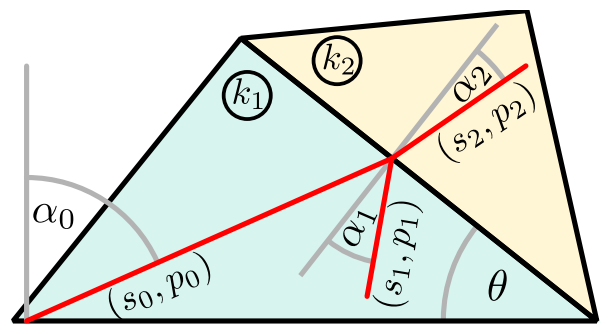

Figure A.12: A ray transports wave energy from half-edge 0 to half-edge 1, where part of the energy is reflected and the rest of the energy is transmitted to half-edge 2 . The direction of the transmitted ray is given by Snell's law.

This generalises to higher order plate crossings, for example, at a T- or Xjunction. Then three or four half-edges are used to describe rays going into each of the three or four plates.

To describe the coordinates used in more detail, let us consider two adjacent polygonal mesh cells as depicted in Fig. A.12. We will now construct the map which takes a ray from one half-edge, say edge 0 , of this polygon to the neighbouring half-edges. In the scenario depicted, the ray hits the edge separating two mesh cells; we denote the half-edge seen by edge 0 as edge 1, which will account for reflection, and the half edge for the transmitted ray as edge 2 . The rays originating from an edge are parametrised by local Birkhoff coordinates $(s, p)$, where $s$ is the arclength along the half-edge and $p=k \sin \alpha$ is the component of the momentum along the edge. The angle $\alpha$ measures the direction of the ray with respect to the normal. The momentum perpendicular to the edge is denoted $p_{\perp}=k \cos \alpha$. The variable $k=2 \pi / \lambda$ is the wavenumber. For triangular mesh cells, the ray tracing map $\Phi_{0 \rightarrow 1}$ from the initial coordinates $\left(s_{0}, p_{0}\right)$ to the final coordinates $\left(s_{1}, p_{1}\right)$ on half-edge 1 is given by

$$
d_{1}\left(s_{1}\right)=d_{0}\left(s_{0}\right) p_{\perp 0} / p_{\perp 1} ; \quad \alpha_{1}=\theta-\alpha_{0} .
$$

Here, $\theta$ is the angle between the two edges, see Fig. A.12, and $d_{i}\left(s_{i}\right)$ is the distance of the point $s_{i}$ from the intersection point between edge 0 and edge 1. The Jacobian of this map is

$$
\mathcal{D} \Phi_{0 \rightarrow 1}=\left[\begin{array}{ll}
\frac{\partial s_{1}}{\partial s_{0}} & \frac{\partial s_{1}}{\partial p_{0}} \\
\frac{\partial p_{1}}{\partial s_{0}} & \frac{\partial p_{1}}{\partial p_{0}}
\end{array}\right]=\left[\begin{array}{cc}
-\frac{p_{\perp 0}}{p_{\perp 1}} & -\frac{D}{p_{\perp 0} p_{\perp 1}} \\
0 & -\frac{p_{\perp 1}}{p_{\perp 0}}
\end{array}\right],
$$

where $D$ denotes the length of the ray from edge 0 to edge 1 . Note that the map $\Phi_{0 \rightarrow 1}$ is phase space volume preserving, that is, $\left|\operatorname{det} \mathcal{D} \Phi_{0 \rightarrow 1}\right|=1$. Fur- 
thermore, note that the wavenumber $k_{1}$ equals $k_{0}$ because both rays travel across the same polygon. Therefore, the wavenumber dependency drops out of the Jacobian for rays parametrised by the same wavenumber. For the transition from half-edge 0 to the half-edge 2 in the neighbouring mesh cell (which may consist of a different material with a different wavenumber), we obtain from Snell's law that $p_{1}=p_{2}$, or $k_{1} \sin \alpha_{1}=k_{2} \sin \alpha_{2}$. By introducing $k_{i}, i=1,2$, in the parametrisation of the relevant phase space coordinates, we again obtain $\left|\operatorname{det} \mathcal{D} \phi_{0 \rightarrow 2}\right|=1$ for the map from half-edge 0 to half-edge 2 and phase space volume preservation follows.

Generalising this procedure, we can define a global phase space on the Surface of Section (SoS) consisting of all half-edges and possible momenta and an associated ray tracing map $\Phi$ from any half-edge to any adjacent half-edge. Technically, this is done using the Frobenius-Perron operator. To simplify notation, we introduce the two-dimensional phase space coordinate $X=(s, p)$, combining both arclength and momentum along all distinct halfedges. Now, let $E$ and $E^{\prime}$ be two adjacent half-edges. The ray density $\varrho(X)$ in the phase space of half-edge $E$ is mapped by the ray tracing map $\Phi$ to the density $\varrho^{\prime}\left(X^{\prime}\right)$ in the phase space of half-edge $E^{\prime}$. In the simplest case without damping or reflection/transmission, we have $\varrho^{\prime}(\Phi(X))=\varrho(X)$ due to phase space volume conservation. Technically this map can be expressed in terms of the integral equation

$$
\varrho^{\prime}\left(X^{\prime}\right)=\{T \varrho\}\left(X^{\prime}\right)=\int \lambda\left(X^{\prime}\right) e^{-\mu D\left(X^{\prime}, X\right)} \delta\left(X^{\prime}-\Phi(X)\right) \varrho(X) \mathrm{d} X .
$$

Here, we have introduced an additional damping factor $e^{-\mu D\left(X^{\prime}, X\right)}$ (with $D\left(X^{\prime}, X\right)$, the distance between the points $X^{\prime}$ and $\left.X\right)$ and a reflection/ transmission factor $\lambda\left(X^{\prime}\right)$. The latter term covers mode conversion and is akin to the coupling loss factors in SEA.

To construct a discrete version of the operator $T$, we project it onto a finite basis by introducing an orthogonal basis system $F_{n}(X)$ with orthogonality relation $\left(F_{n}, F_{m}\right)=\left\|F_{n}\right\|^{2} \delta_{n, m}$ and $\left\|F_{n}\right\|^{2}=\left(F_{n}, F_{n}\right)$. The inner product is defined as

$$
(u, v)=\int u(X) v(X) \mathrm{d} X,
$$

where $u$ and $v$ are two functions on the phase space of all edges and $-k \leq$ $p \leq k$. Note that the wavenumber $k$ may differ on different edges or for different modes. We can now express the relation between an initial density $\varrho(X)$ and the corresponding propagated density $\varrho^{\prime}\left(X^{\prime}\right)=(T \varrho)\left(X^{\prime}\right)$ in this 
basis as

$$
\varrho(X)=\sum_{n} f_{n} F_{n}(X), \quad \varrho^{\prime}\left(X^{\prime}\right)=\sum_{n^{\prime}} f_{n^{\prime}}^{\prime} F_{n^{\prime}}\left(X^{\prime}\right)
$$

where

$$
f_{n^{\prime}}^{\prime}=\sum_{n} T_{n^{\prime} n} f_{n}
$$

Here, the matrix representation of the Frobenius-Perron operator $T$ is given by

$$
T_{n^{\prime} n}=\frac{1}{\left(F_{n^{\prime}}, F_{n^{\prime}}\right)}\left(F_{n^{\prime}}, T F_{n}\right)
$$

The matrix elements can be evaluated using the inner product

$$
\begin{aligned}
\left(F_{n^{\prime}}, T F_{n}\right) & =\int F_{n^{\prime}}\left(X^{\prime}\right)\left\{T F_{n}\right\}\left(X^{\prime}\right) \mathrm{d} X^{\prime} \\
& =\iint F_{n^{\prime}}(X) \lambda\left(X^{\prime}\right) e^{-\mu D\left(X^{\prime}, X^{\prime}\right)} \delta\left(X^{\prime}-\Phi(X)\right) F_{n}(X) \mathrm{d} X \mathrm{~d} X^{\prime} \\
& =\int \lambda(\Phi(X)) e^{-\mu D(\Phi(X), X)} F_{n^{\prime}}(\Phi(X)) F_{n}(X) \mathrm{d} X .
\end{aligned}
$$

The choice of basis employed here is piecewise constant functions in position space and Legendre polynomials in momentum space, that is,

$$
F_{(b, \beta)}(s, p)=\frac{1}{\sqrt{A_{b} k_{b}}} \mathbb{1}_{b}(s) P_{\beta}\left(\frac{p}{k_{b}}\right) ; \quad\left(F_{(b, \beta)}, F_{(\tilde{b}, \tilde{\beta})}\right)=\frac{2}{2 \beta+1} \delta_{b, \tilde{b}} \delta_{\beta, \tilde{\beta}},
$$

where $\mathbb{1}_{b}$ is the characteristic function of edge $b$ (that is, $\mathbb{1}_{b}(s)=1$ for $s$ on edge $b$ and 0 elsewhere) and $A_{b}$ is the length of edge $b$. For an implementation using higher order basis functions in position space, see [12]. The multiindex $\mathbf{n} \equiv(b, \beta)$ combines the index of the edge $b$ with the index $\beta$, which corresponds to the degree of the Legendre polynomial. The matrix elements in this basis read

$$
\begin{aligned}
T_{\mathbf{n}^{\prime} \mathbf{n}}= & \frac{2 \beta^{\prime}+1}{2 \sqrt{A_{b^{\prime}} A_{b} k_{b^{\prime}} k_{b}}} \\
& \int_{0}^{A_{b}} \int_{-k_{b}}^{+k_{b}} \lambda\left(x^{\prime}\right) e^{-\mu D\left(s^{\prime}, s\right)} \mathbb{1}_{b^{\prime}}\left(s^{\prime}\right) \mathbb{1}_{b}(s) P_{\beta^{\prime}}\left(p^{\prime} / k_{b^{\prime}}\right) P_{\beta}\left(p / k_{b}\right) \mathrm{d} p \mathrm{~d} s
\end{aligned}
$$

or in the angular variable $\alpha$ (with $p=k_{b} \sin \alpha$ and $\sin \alpha^{\prime}=\left(k_{b} / k_{b^{\prime}}\right) \sin (\theta-\alpha)$ 


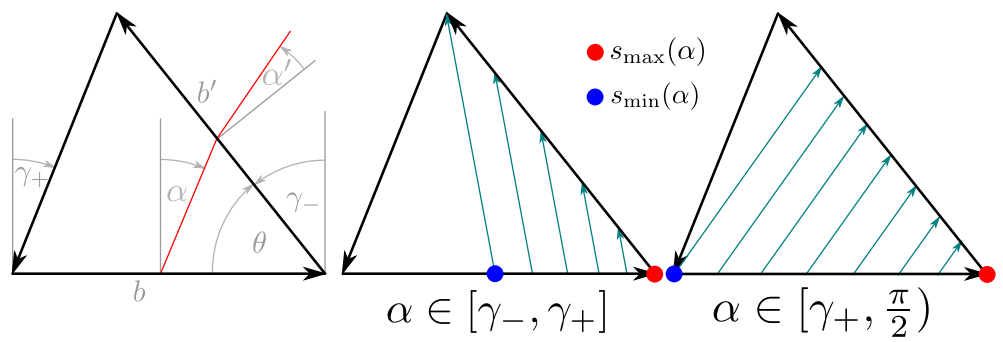

Figure A.13: An illustration of the geometry underlying the position integral, showing is the transmitted ray. The target half-edge $b^{\prime}$ corresponds here to the upper right side of the triangle.

according to Snell's law) we have

$$
\begin{aligned}
T_{\mathbf{n}^{\prime} \mathbf{n}} & =\frac{2 \beta^{\prime}+1}{2 \sqrt{A_{b^{\prime}} A_{b}}} \sqrt{\frac{k_{b}}{k_{b^{\prime}}}} \\
& \int_{0}^{A_{b}} \int_{-\pi / 2}^{+\pi / 2} \lambda\left(x^{\prime}\right) e^{-\mu D\left(s^{\prime}, s\right)} \mathbb{1}_{b^{\prime}}\left(s^{\prime}\right) \mathbb{1}_{b}(s) P_{\beta^{\prime}}\left(\sin \alpha^{\prime}\right) P_{\beta}(\sin \alpha) \cos \alpha \mathrm{d} \alpha \mathrm{d} s .
\end{aligned}
$$

In the following, we assume that $\lambda\left(x^{\prime}\right)$ is only a function of the angle. Then the calculation of the double integral simplifies as the position integral may be evaluated analytically, reducing the computation to a one-dimensional numerical integral over the angular variable:

$$
T_{\mathbf{n}^{\prime} \mathbf{n}}=\frac{2 \beta^{\prime}+1}{2 \sqrt{A_{b^{\prime}} A_{b}}} \sqrt{\frac{k_{b}}{k_{b^{\prime}}}} \int_{-\pi / 2}^{+\pi / 2} \lambda\left(\alpha^{\prime}\right) h(\mu, \alpha) P_{\beta^{\prime}}\left(\sin \alpha^{\prime}\right) P_{\beta}(\sin \alpha) \cos \alpha \mathrm{d} \alpha .
$$

The position integral

$$
h(\mu, \alpha)=\int_{0}^{A_{b}} e^{-\mu D\left(s^{\prime}, s\right)} \mathbb{1}_{b^{\prime}}\left(s^{\prime}\right) \mathbb{1}_{b}(s) \mathrm{d} s=\int_{s_{\min }(\alpha)}^{s_{\max }(\alpha)} e^{-\mu D\left(s^{\prime}, s\right)} \mathrm{d} s
$$

can be evaluated analytically [6], see Fig. A.13. For zero damping, that is, $\mu=0$, we have

$$
h(0, \alpha)= \begin{cases}0 & \text { for } \alpha \in\left[-\frac{\pi}{2}, \gamma_{-}\right] \\ A_{b^{\prime}} \frac{\cos \alpha_{1}}{\cos \alpha} & \text { for } \alpha \in\left[\gamma_{-}, \gamma_{+}\right] \\ A_{b} & \text { for } \alpha \in\left[\gamma_{+},+\frac{\pi}{2}\right]\end{cases}
$$


with $\gamma_{ \pm}$corresponding to the exterior angles of the triangular mesh cell depicted in Fig. A.13. Here $\alpha_{1}=\theta-\alpha$ is the angle at which the ray arrives at the receiving edge without applying Snell's law. One can use the position integral $h(0, \alpha)$ for zero damping given in (A.3) to express the position integral $h(\mu, \alpha)$ for non-zero values of the damping. For $\mu \neq 0$, one obtains

$$
h(\mu, \alpha)=\frac{1-e^{-\tilde{\mu} h(0, \alpha)}}{\tilde{\mu}} \quad \text { with } \quad \tilde{\mu}=\mu \frac{\sin |\theta|}{\cos \alpha_{1}} .
$$

The function $h(\mu, \alpha)$ is a piecewise smooth function of the angle $\alpha$. The numerical integration over the angular variable $\alpha$ is now split into three sub-intervals according to Eq. (A.3). If the reflection/transmission coefficient $\lambda\left(\alpha^{\prime}\right)$ is smooth in each interval, then the integrand is smooth and the numerical integral can be calculated using Clenshaw-Curtis integration (or similar schemes) to high precision. If $\lambda\left(\alpha^{\prime}\right)$ also has singularities, more care has to be taken when performing the numerical integration.

Having determined the transfer operator $T$, the stationary phase space density $\varrho_{\infty}$ on the boundaries of each of the mesh cells is obtained by iteration, that is

$$
\varrho_{\infty}=\sum_{n=0}^{\infty} T^{n} \varrho_{0}=(1-T)^{-1} \varrho_{0} .
$$

Here, $\varrho_{0}$ is the initial source density projected onto the mesh boundaries as discussed in Appendix C.

In this work we assume that the structure is assembled from thin plates. Elements of this type support three wave modes: longitudinal and shear waves, which are both in-plane modes with a linear dispersion relation, and bending waves, which are an out-of-plane mode with a quadratic dispersion relation. Each of these three modes is described by its own ray density; technically this is done by enlarging the multi-index $\mathbf{n}$ to $\mathbf{n} \equiv(m, b, \beta)$ by introducing a mode number index $m$. At an edge, the three different modes can be converted into one other. The conversion coefficients are included as part of the reflection/transmission coefficients $\lambda\left(X^{\prime}\right)$ and are given by the scattering matrix derived in [9]. Fig. C.15 shows as an example the results for an L-joint consisting of two plates fixed at a right angle. The elements of the scattering matrix $\mathcal{S}_{m n}^{a b}$ are plotted where the indices $m, n$ are the wave modes $P, S$ or $B$ and $a, b=1,2$ represent the two plates. The plates in the example have identical properties and the frequency is chosen such that $k_{\mathrm{L}} / k_{\mathrm{B}}=0.3$. The modulus squared of the scattering matrix element $\mathcal{S}_{m n}^{a b}$ are the reflection $(a=b)$ and transmission $(a \neq b)$ coefficient. 


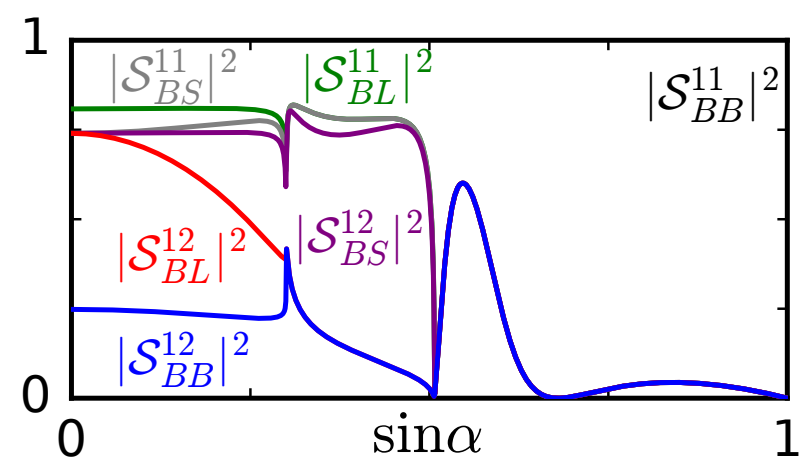

Figure A.14: Transmission and reflection coefficients for two plates at a $90^{\circ}$ angle. The different coefficients are added up, so $\left|\mathcal{S}_{B L}^{12}\right|^{2}$ is the distance from the red curve to the blue one. $\alpha$ is the angle of the incoming ray.

This allows DEA to take curvature, plate intersections and varying material parameters into account. In this work we are using a hysteretic damping model, which replaces Young's modulus $E$ with the complex value $E_{c}=E(1+i \eta)$. Here, $\eta$ is the hysteretic damping parameter. The corresponding decay in the energy density of a plane wave across a distance $D$ (in the propagation direction) is $e^{-\eta k_{\mathrm{L} / \mathrm{S}} D}$ for longitudinal and shear waves, and $e^{-\eta k_{\mathrm{B}} D / 2}$ for bending waves where $k_{\mathrm{L} / \mathrm{S} / \mathrm{B}}$ is the corresponding wavenumber. It follows that the damping parameters for DEA are $\mu_{\mathrm{L} / \mathrm{S}}=\eta k_{\mathrm{L} / \mathrm{S}}$ and $\mu_{\mathrm{B}}=\eta k_{\mathrm{B}} / 2$.

Remark: The DEA formulation as described here and later in Appendix $\mathrm{C}$ can be simplified by multiplying the vector of expansion coefficients $f_{n}$ by $\sqrt{k_{n}}$. If done consistently, one can drop the $\sqrt{k_{n}}$ prefactors in the expressions for the matrix elements, Eq. (A.2) and later Eq. (D.2), as well as in the relation for the spatial or interior density (C.4) and the source densities (C.8) and (C.10). However, the $\sqrt{k_{n}}$ factors must remain present in the source strength of the point force given in Eq. (C.12) and in the weighting factors for the RBEs (D.3).

\section{Appendix B. Transformation of Surface of Section coordinates to bulk coordinates}

In the previous section, we have worked with a ray density on a SoS formed by the union of all edges of a polygonal mesh and associated momenta. We will now show how this boundary density can be related to a density in the interior of each mesh cell. The first step is to lift the density from 
the SoS coordinates $X=(s, p)$ to the full four dimensional phase space coordinates using a $4 \mathrm{D}$ coordinate transformation. We introduce the coordinate system $\mathbf{x} \equiv\left(q_{\|}, p_{\|}, q_{\perp}, p_{\perp}\right)$ for the full phase space at an edge with $q_{\|} \equiv s$, $p \equiv p_{\|}$and $\left(q_{\perp}, p_{\perp}\right)$ are the position and momentum perpendicular to the edge. The SoS coordinates $(s, p)$ can be augmented to full four dimensional phase space coordinates by introducing the time $t$ (along the propagation direction of the ray in question) and the frequency $\omega$ (equivalent to the energy $E$ in classical mechanics). Therefore, the desired coordinate transformation is from the coordinates $(s, p, t, \omega)$ to coordinates $\left(q_{\|}, p_{\|}, q_{\perp}, p_{\perp}\right)$. To relate the transformation to the underlying ray-dynamics, we must give a relation between the frequency $\omega$ and the momentum vector ${ }^{1}\left(p_{\|}, p_{\perp}\right)$. This is done by introducing a Hamilton function $H$ given in the form of the dispersion relation

$$
H\left(q_{\|}, p_{\|}, q_{\perp}, p_{\perp}\right)=\alpha k^{2 \beta}=\omega \quad \text { with } \quad k=\sqrt{p_{\|}^{2}+p_{\perp}^{2}},
$$

which is valid for isotropic and homogeneous (translation invariant) systems. Here, $k$ is the wavenumber and $\beta=\frac{1}{2}$ for linear dispersion relations (longitudinal and shear modes), or $\beta=1$ for quadratic dispersion relations (bending modes). The equations of motion are then

$$
\dot{\mathbf{x}}=\left(\begin{array}{c}
\frac{\partial H}{\partial p_{\|}} \\
-\frac{\partial H}{\partial q_{\|}} \\
\frac{\partial H}{\partial p_{\perp}} \\
-\frac{\partial H}{\partial q_{\perp}}
\end{array}\right)=\frac{2 \beta \omega}{k^{2}}\left(\begin{array}{c}
p_{\|} \\
0 \\
p_{\perp} \\
0
\end{array}\right) .
$$

For homogeneous and isotropic systems, the modulus of the velocity equals the group velocity $v_{g}=\frac{\partial \omega}{\partial k}$ and is

$$
|\dot{\mathbf{x}}|=v_{g}=2 \beta \frac{\omega}{k}=2 \beta v_{p h},
$$

where we have introduced the phase velocity $v_{p h}=\omega(k) / k$. Note, that for longitudinal/shear waves $v_{p h}=v_{g}=\alpha$ (linear dispersion relation). For bending waves (quadratic dispersion relation), we have that the group velocity $v_{g, \mathrm{~B}}=2 v_{p h}(k)$ with $v_{p h}(k)=\alpha k$. The parameter $\alpha$ is given by material constants.

\footnotetext{
${ }^{1}$ Note that the momentum vector is equivalent to the wave vector, in the same way as the Hamiltonian $H$ is equivalent to the frequency $\omega$.
} 
The linearised four dimensional coordinate transformation $\Omega$ is now given as

$$
\Omega: \quad\left[\begin{array}{c}
s \\
p \\
t \\
\omega
\end{array}\right] \mapsto\left[\begin{array}{c}
q_{\|} \\
p_{\|} \\
q_{\perp} \\
p_{\perp}
\end{array}\right]+\frac{2 \beta \omega t}{k^{2}}\left(\begin{array}{c}
p_{\|} \\
0 \\
p_{\perp} \\
0
\end{array}\right)
$$

with Jacobian matrix

$$
\mathcal{D} \Omega=\left[\begin{array}{cccc}
1 & 0 & 2 \beta \omega p_{\|} /\left(p_{\|}^{2}+p_{\perp}^{2}\right) & 0 \\
0 & 1 & 0 & 0 \\
0 & 0 & 2 \beta \omega p_{\perp} /\left(p_{\|}^{2}+p_{\perp}^{2}\right) & 0 \\
0 & -p_{\|} / p_{\perp} & 0 & \left(p_{\|}^{2}+p_{\perp}^{2}\right) /\left(2 \beta \omega p_{\perp}\right)
\end{array}\right] .
$$

Here, we have used that

$$
\frac{\partial p_{\perp}(s, p, t, \omega)}{\partial p_{\|}}=-\frac{p_{\|}}{p_{\perp}} \quad \text { and } \quad \frac{\partial p_{\perp}(s, p, t, \omega)}{\partial \omega}=\frac{p_{\|}^{2}+p_{\perp}^{2}}{2 \beta \omega p_{\perp}},
$$

which can be derived from (B.1). Note that $\mathcal{D} \Omega$ is symplectic and in particular, $\operatorname{det} \mathcal{D} \Omega=1$. That means that the density at the new coordinates $\left(q_{\|}, p_{\|}, q_{\perp}, p_{\perp}\right)$ is simply the density in the old coordinates evaluated at the corresponding point $(s, p, t, \omega)$. To lift the density $\varrho(s, p)$ to the $4 \mathrm{D}$ coordinate system $(s, p, t, \omega)$ we write

$$
\varrho_{4 D}(s, p, t, \omega)=\varrho(s, p) \omega_{0} \delta\left(\omega-\omega_{0}\right),
$$

where we make use of the stationarity of the solution for fixed frequency $\omega=\omega_{0}$. The additional factor $\omega_{0}$ is introduced for dimensionality reasons. In full phase space coordinates, we obtain

$$
\varrho_{4 D}\left(q_{\|}, p_{\|}, q_{\perp}, p_{\perp}\right)=\varrho\left(q_{\|}, p_{\|}\right) \omega_{0} \delta\left(\omega(k)-\omega_{0}\right),
$$

with $\omega(k)=H\left(p_{\|}, p_{\perp}\right)$ from (B.1). Writing the density directly on the manifold $k=$ const, we obtain (using the group velocity $v_{g}(k)=\frac{\partial \omega}{\partial k}$ ) that

$$
\varrho_{4 D}\left(q_{\|}, p_{\|}, q_{\perp}, p_{\perp}\right)=\varrho\left(q_{\|}, p_{\|}\right) \frac{\omega_{0}}{v_{g, 0}} \delta\left(k-k_{0}\right),
$$

with $k_{0}$ related to $\omega_{0}$ through the dispersion relation (B.1).

In some cases, we need $p_{\perp}$ as the argument inside the delta function. Applying the substitution rule once more, we get

$$
\varrho_{4 D}\left(q_{\|}, p_{\|}, q_{\perp}, p_{\perp}\right)=\varrho\left(q_{\|}, p_{\|}\right) \frac{\omega_{0}}{v_{g, 0}} \frac{k_{0}}{p_{\perp, 0}} \delta\left(p_{\perp}-p_{\perp, 0}\right)
$$

with $p_{\perp, 0}=\sqrt{k_{0}^{2}-p_{\|}^{2}}$. 


\section{Appendix C. Energy density in the interior}

\section{Appendix C.1. Spatial energy density}

The spatial ray density $\varrho_{\mathrm{sp}}(\mathbf{r})$ at a point $\mathbf{r}=(x, y)$ in the interior of a mesh cell is defined as the integral of the phase space density over all momentum components $\mathbf{p}=\left(p_{x}, p_{y}\right)$, that is,

$$
\varrho_{\mathrm{sp}}(\mathbf{r})=\int \varrho_{4 D}(\mathbf{r}, \mathbf{p}) \mathrm{d} \mathbf{p} .
$$

The quantity $\varrho_{4 \mathrm{D}}(\mathbf{r}, \mathbf{p})$ at a point $\mathbf{r}$ in the interior of a polygon is directly related to the phase space density on the edges of the polygon. Denoting the intersection of the ray emanating from $\mathbf{r}$ in direction $-\mathbf{p}$ with the boundary of the polygon as $\mathbf{r}_{\mathrm{bd}}(\mathbf{r}, \mathbf{p})$, one obtains

$$
\varrho_{\mathrm{sp}}(\mathbf{r})=\int \varrho_{4 \mathrm{D}}(\mathbf{r}, \mathbf{p}) \mathrm{d} \mathbf{p}=\int e^{-\mu\left|\mathbf{r}-\mathbf{r}_{\mathrm{bd}}\right|} \varrho_{4 \mathrm{D}}\left(\mathbf{r}_{\mathrm{bd}}(\mathbf{r}, \mathbf{p}), \mathbf{p}\right) \mathrm{d} \mathbf{p} .
$$

Writing the integral with respect to $\mathbf{p}$ in polar coordinates $(k, \phi)$ leads to $\mathbf{p}=(k \cos \phi, k \sin \phi)$ and $\mathrm{d} \mathbf{p}=k \mathrm{~d} \phi \mathrm{d} k$, then applying Eq. (B.3) gives

$$
\begin{aligned}
\varrho_{\mathrm{sp}}(\mathbf{r}) & =\int_{0}^{\infty} \int_{0}^{2 \pi} e^{-\mu\left|\mathbf{r}-\mathbf{r}_{\mathrm{bd}}\right|} \varrho(s(\mathbf{r}, \phi), k \sin \alpha(\mathbf{r}, \phi)) \frac{\omega_{0}}{v_{g, 0}} \delta\left(k-k_{0}\right) k \mathrm{~d} \phi \mathrm{d} k \\
& =\frac{\omega_{0} k_{0}}{v_{g, 0}} \int_{0}^{2 \pi} e^{-\mu\left|\mathbf{r}-\mathbf{r}_{\mathrm{bd}}\right|} \varrho\left(s(\mathbf{r}, \phi), k_{0} \sin \alpha(\mathbf{r}, \phi)\right) \mathrm{d} \phi .
\end{aligned}
$$

Here, $\phi$ is the polar angle parametrising trajectories approaching $\mathbf{r}$, and $s(\mathbf{r}, \phi)$ and $\alpha(\mathbf{r}, \phi)$ are the position and angle at which rays hit the boundary, respectively. In the last equation, we could also write the pre-factor as $\omega_{0} k_{0} v_{g, 0}^{-1}=\omega_{0}^{2} v_{p h, 0}^{-1} v_{g, 0}^{-1}$. This form is closer to the result from Eqs. (27-33) in [13], where the special case $v_{g}=v_{p h}$ is considered.

After a change of variables from $\phi$ to $s$, see Fig. C.15, one obtains

$$
\varrho_{\mathrm{sp}}(\mathbf{r})=\frac{\omega_{0} k_{0}}{v_{g, 0}} \int e^{-\mu D(s, \mathbf{r})} \varrho\left(s, k_{0} \sin \alpha(s, \mathbf{r})\right) \frac{\cos \alpha(s, \mathbf{r})}{D(s, \mathbf{r})} \mathrm{d} s,
$$

where $s$ is the arc length around the boundary of the polygonal mesh cell containing $\mathbf{r}$ and $D(s, \mathbf{r})=\left|\mathbf{r}-\mathbf{r}_{\mathrm{bd}}(s)\right|$ is the distance of the point $\mathbf{r}$ from the point on the boundary. After inserting a basis expansion of the form

$$
\varrho(s, p)=\sum_{b, \beta} \frac{f_{b, \beta}}{\sqrt{A_{b} k_{b}}} \mathbb{1}_{b}(s) P_{\beta}\left(p / k_{b}\right)
$$




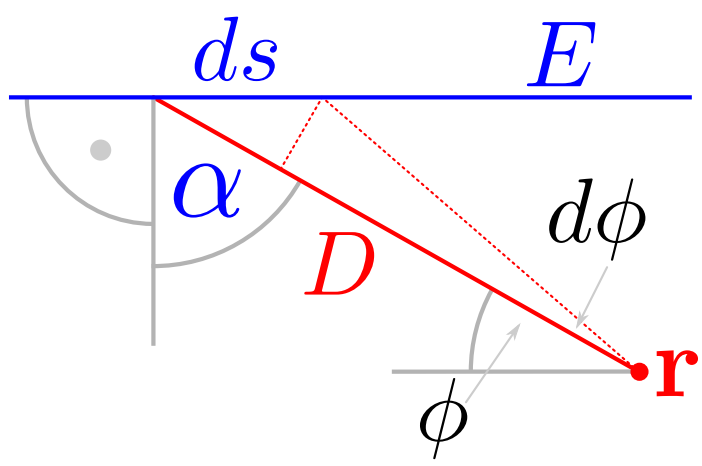

Figure C.15: Coordinate transformation from angle to arc-length coordinates.

into Eq. (C.3), the spatial density can be written as

$$
\varrho_{\mathrm{sp}}(\mathbf{r})=\frac{\omega_{0}}{v_{g, 0}} \sum_{b, \beta} f_{b, \beta} \sqrt{\frac{k_{b}}{A_{b}}} \int e^{-\mu D(s, \mathbf{r})} \mathbb{1}_{b}(s) P_{\beta}(\sin \alpha(s, \mathbf{r})) \frac{\cos \alpha(s, \mathbf{r})}{D(s, \mathbf{r})} \mathrm{d} s
$$

where the sum includes all half-edges of the polygonal mesh cell containing the point $\mathbf{r}$. For these half-edges we have $k_{0}=k_{b}$.

\section{Appendix C.2. Momentum density}

As well as considering wave energy transport and the calculation of wave energy densities, we may also consider the momentum transport properties of our wave system. In order to get a quantitative measure of the momentum transport, we define the momentum density $\varrho_{\text {mom }}(\mathbf{r})$ at a point $\mathbf{r}=(x, y)$ (in the interior of a mesh cell) as the integral of the momentum vector $\mathbf{p}=\left(p_{x}, p_{y}\right)$ weighted by the phase space density, that is

$$
\varrho_{\mathrm{mom}}(\mathbf{r})=\int \mathbf{p} \varrho_{4 D}(\mathbf{r}, \mathbf{p}) \mathrm{d} \mathbf{p} .
$$

Following the steps of the previous section, we can write

$$
\varrho_{\text {mom }}(\mathbf{r})=\frac{\omega_{0} k_{0}^{2}}{v_{g, 0}} \int \frac{\mathbf{D}(s, \mathbf{r})}{D(s, \mathbf{r})} e^{-\mu D(s, \mathbf{r})} \varrho\left(s, k_{0} \sin \alpha(s, \mathbf{r})\right) \frac{\cos \alpha(s, \mathbf{r})}{D(s, \mathbf{r})} \mathrm{d} s .
$$

Here the vector $\mathbf{D}(s, \mathbf{r})=\mathbf{r}-\mathbf{r}_{\mathrm{bd}}$ indicates the propagating direction and hence the momentum of the ray. In terms of our basis, the momentum 
density reads

$$
\begin{array}{r}
\varrho_{\text {mom }}(\mathbf{r})=\frac{\omega_{0} k_{0}}{v_{g, 0}} \sum_{b, \beta} f_{b, \beta} \sqrt{\frac{k_{b}}{A_{b}}} \int \frac{\mathbf{D}(s, \mathbf{r})}{D(s, \mathbf{r})} e^{-\mu D(s, \mathbf{r})} \mathbb{1}_{b}(s) \\
P_{\beta}(\sin \alpha(s, \mathbf{r})) \frac{\cos \alpha(s, \mathbf{r})}{D(s, \mathbf{r})} \mathrm{d} s .
\end{array}
$$

The magnitude of the momentum density vector varies greatly across the structure. Therefore in order to visualize the momentum density, we adjust the length of the plotted vector to $\log \left(\left|\varrho_{\text {mom }}(\mathbf{r})\right| / k_{\text {mom,ref }}\right)$ where $k_{\text {mom,ref }}$ is a fixed reference scale. The direction of the plotted vector is unchanged.

\section{Appendix C.3. Line source}

Next we turn to a description of the source terms. We start by modelling a source along an infinite edge $E_{b_{0}}$ radiating out in the direction $\phi_{0}$ with strength $R_{0}$. The initial phase space density on the edge for this scenario is given by

$$
\varrho_{0}(s, p)=R_{0} \omega_{0}^{-1} \cos \phi_{0} \mathbb{1}_{b_{0}}(s) \delta\left(p-p_{0}\right)
$$

with tangential component $p_{0}=k_{0} \sin \phi_{0}$. The source strength $R_{0}$ plays the role of an intensity, that is, the energy per unit time flowing through a unit line element perpendicular to the direction of the source. Starting from Eq. (B.4), we get the four dimensional phase space density as

$$
\varrho_{4 D, 0}(\mathbf{r}, \mathbf{p})=R_{0} v_{g, 0}^{-1} \delta\left(\mathbf{p}-\mathbf{p}_{0}\right)
$$

with $\mathbf{p}_{0}=\left(k_{0} \sin \phi_{0}, k_{0} \cos \phi_{0}\right)$ and a constant spatial density

$$
\varrho_{\mathrm{sp}, 0}(\mathbf{r})=R_{0} v_{g, 0}^{-1} .
$$

We obtain, as expected, that the intensity equals the energy density times the group velocity. Expanding the source density into the basis components, one obtains

$$
\varrho_{0}(X)=\sum_{n} f_{0, n} F_{n}(X) \quad \text { with } \quad f_{0, n}=\frac{\left(\varrho_{0}, F_{n}\right)}{\left(F_{n}, F_{n}\right)} .
$$

In our specific basis, this reads

$$
f_{0, \mathbf{n}}=R_{0} \omega_{0}^{-1} \cos \phi_{0} \delta_{b, b_{0}} \sqrt{\frac{A_{b_{0}}}{k_{b_{0}}}}\left(\frac{2 \beta+1}{2}\right) P_{\beta}\left(\sin \phi_{0}\right)
$$

with $\mathbf{n}=(b, \beta)$ and $p_{0}=k_{b_{0}} \sin \phi_{0}$. 


\section{Appendix C.4. Point source}

We now consider the case of a point source in the interior of a mesh cell at a position $\mathbf{r}_{0}$. The source strength is again denoted as $R_{0}$ and can be interpreted as the energy which flows out of the source per unit time. The $4 \mathrm{D}$ energy density is then given as

$$
\varrho_{0,4 \mathrm{D}}(\mathbf{r}, \mathbf{p})=\frac{R_{0}}{2 \pi v_{g, 0}} \frac{e^{-\mu\left|\mathbf{r}-\mathbf{r}_{0}\right|}}{\left|\mathbf{r}-\mathbf{r}_{0}\right|} \delta\left(\mathbf{p}-k_{0} \frac{\mathbf{r}-\mathbf{r}_{0}}{\left|\mathbf{r}-\mathbf{r}_{0}\right|}\right)
$$

and the spatial energy density is

$$
\varrho_{\mathrm{sp}, 0}(\mathbf{r})=\frac{R_{0}}{2 \pi v_{g, 0}} \frac{e^{-\mu\left|\mathbf{r}-\mathbf{r}_{0}\right|}}{\left|\mathbf{r}-\mathbf{r}_{0}\right|}
$$

According to Eq. (B.4), the corresponding 2D boundary energy density is given as

$$
\varrho_{0}(s, p)=\frac{R_{0}}{2 \pi \omega_{0}} \frac{e^{-\mu D(s, \mathbf{r})}}{D(s, \mathbf{r})} \delta\left(p-k_{0} \sin \alpha(s, \mathbf{r})\right) \cos \alpha(s, \mathbf{r}) .
$$

If one neglects damping by setting $\mu=0$ and calculates the energy flowing through the boundary per unit time, one gets $R_{0}$ as expected. Technically, this is done by integrating the product of the energy density (C.9) and the group velocity $v_{g, 0}$ around a circle with centre $\mathbf{r}_{0}$. Next, we project the source density onto our basis as follows

$$
\varrho_{0}(X)=\sum_{n} f_{0, n} F_{n}(X) \quad \text { with } \quad f_{0, n}=\frac{\left(\varrho_{0}, F_{n}\right)}{\left(F_{n}, F_{n}\right)} .
$$

In our specific basis, this reads

$$
f_{0, \mathbf{n}}=\frac{R_{0}}{2 \pi \omega_{0}} \sqrt{\frac{1}{A_{b} k_{b}}}\left(\frac{2 \beta+1}{2}\right) \int \lambda(\alpha(s)) e^{-\mu D(s, \mathbf{r})} P_{\beta}(\sin \alpha(s)) \frac{\cos \alpha(s)}{D(s, \mathbf{r})} \mathrm{d} s
$$

with $\mathbf{n}=(b, \beta)$. Here the reflection/transmission coefficient $\lambda(\alpha(s))$ accounts for a possible change in material parameters between the polygon containing the source and the polygon belonging to $f_{0, \mathbf{n}}$. The angle $\alpha(s)$ takes Snell's law into account.

\section{Appendix C.5. Point excitation of a thin plate}

Consider an infinitely extended thin plate which is excited by a timeharmonic point force of strength $F_{0}$ acting perpendicular to the plate. The equation of motion for the resulting bending wave $u(\mathbf{r})$ is

$$
h \varrho_{p} \ddot{u}(\mathbf{r})+\mathfrak{D} \Delta^{2} u(\mathbf{r})=F_{0} e^{i \omega t} \delta\left(\mathbf{r}-\mathbf{r}_{0}\right)
$$


with $\mathbf{r}, \mathbf{r}_{0} \in \mathbb{R}^{2}$. Here, $h$ is the plate thickness, $\varrho_{p}$ is the (volume-)density of the plate and $\mathfrak{D}=\frac{h^{3}}{12} \frac{E}{1-\nu^{2}}$ is the so-called the flexural rigidity (also known as the bending stiffness) with Young's modulus $E$ and Poisson ratio $\nu$. Using a time-harmonic ansatz gives the biharmonic Helmholtz equation

$$
\left[\Delta^{2}-k^{4}\right] u=\frac{F_{0} k^{4}}{h \varrho_{p} \omega^{2}} \delta\left(\vec{r}-\vec{r}_{0}\right)
$$

with dispersion relation $h \varrho_{p} \omega^{2} / \mathfrak{D}=k^{4}$. The Green's function solving (C.11) can be written as a combination of the Green's functions for the Helmholtz equation and the modified Helmholtz equation using the relation

$$
\left(\Delta^{2}-k^{4}\right)^{-1}=\frac{1}{2 k^{2}}\left[\left(\Delta-k^{2}\right)^{-1}-\left(\Delta+k^{2}\right)^{-1}\right] .
$$

The Green's function $G_{\text {Hel }}$ of the 2D Helmholtz equation with $r=\left|\mathbf{r}-\mathbf{r}_{0}\right|$ is given by

$$
G_{\text {Hel }}(r)=-\frac{i}{4} H_{0}^{(1)}(r k),
$$

and satisfies $\left[\Delta+k^{2}\right] G_{\mathrm{Hel}}(r)=\delta\left(\mathbf{r}-\mathbf{r}_{0}\right)$. Hence, one obtains the Green's function $G_{\text {bih }}$ for the biharmonic Helmholtz equation as

$$
\left[\Delta^{2}-k^{4}\right] G_{\mathrm{bih}}=\delta\left(\mathbf{r}-\mathbf{r}_{0}\right) ; \quad G_{\mathrm{bih}}(\mathbf{r})=\frac{1}{2 k^{2}}\left[\frac{i}{4} H_{0}^{(1)}(r k)-\frac{1}{2 \pi} K_{0}(r k)\right] .
$$

The asymptotic behaviour for $r \rightarrow \infty$ is then given by

$$
G_{\mathrm{bih}}(\mathbf{r}) \sim \frac{1}{8 k^{2}} \sqrt{\frac{2}{\pi r k}}\left[e^{i(r k-\pi / 4)}-e^{-r k}\right]
$$

and is equivalent to the high frequency asymptotics as $k \rightarrow \infty$. Therefore the propagating part of the solution of the original problem, Eq. (C.11), is given as

$$
u(\mathbf{r})=\frac{i F_{0} k^{2}}{8 h \varrho_{p} \omega^{2}} \sqrt{\frac{2}{\pi r k}} e^{i r k-i \pi / 4}
$$

with a related energy density $e=\varrho_{p} h|\dot{u}|^{2} / 2$ of the form

$$
e=\frac{F_{0}^{2} k^{3}}{32 h \varrho_{p} \omega^{2}} \frac{1}{2 \pi r} .
$$

Comparing with Eq. (C.9) and using $v_{g}=2 \frac{\omega}{k}$, one obtains

$$
R_{0}=\frac{F_{0}^{2} k^{2}}{16 h \varrho_{p} \omega} .
$$



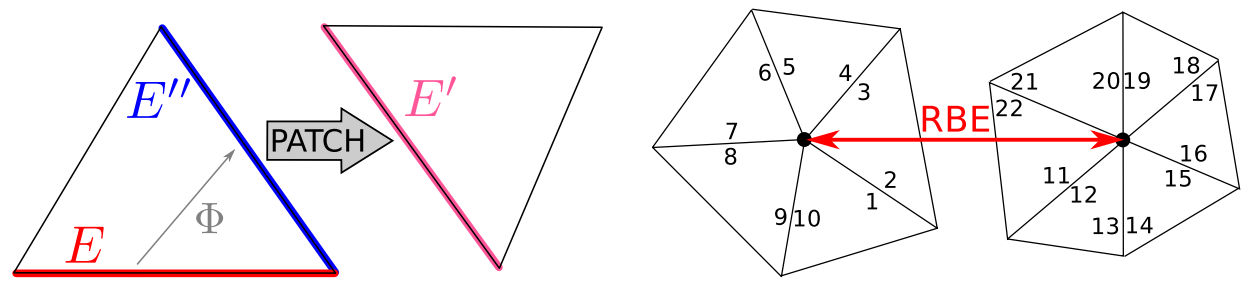

Figure D.16: Left: An illustration of the RBE patch elements in DEA; here edge $E^{\prime \prime}$ is connected to edge $E^{\prime}$ with an RBE and energy flows from edge $E$ to edge $E^{\prime}$. Right: DEA patch elements in a situation where many edges are connected by RBEs.

\section{Appendix D. Implementation of Rigid Body Elements (RBEs) in DEA}

To illustrate RBE patch modeling in DEA as discussed in Sec. 3.2, we consider the following simplified scenario of having three edges, $E, E^{\prime \prime}$ and $E^{\prime}$ as shown in the left part of Fig. D.16. The edges $E$ and $E^{\prime \prime}$ are neighbours and energy flows in the same way as in ordinary DEA. However, we now assume that $E^{\prime \prime}$ and $E^{\prime}$ are connected via an RBE and can thus exchange energy even though they do not have a common interface; these connections need to be handled by RBE patches. In the transfer operator, we now create a connection between edges $E$ and $E^{\prime}$ without referring to the edge $E^{\prime \prime}$ directly. The edge $E^{\prime \prime}$ is called the base and the edge $E^{\prime}$ is called the target.

The RBE patch element as introduced in Sec. 3.2 is a diffusive element. To model this mathematically, we replace the sharp delta function in the transfer operator Eq. (A.1) with a general distribution

$$
\{T \varrho\}\left(X^{\prime}\right)=\int \Xi\left(X^{\prime}, X\right) \varrho(X) \mathrm{d} X .
$$

The final distribution should not depend on $X^{\prime}$, i.e. it should be equidistributed in phase space. We enforce the condition that only rays which hit the edge $E^{\prime \prime}$ contribute. When a ray hits $E^{\prime \prime}$ we require energy conservation for $E^{\prime}$, that is,

$$
\int \Xi\left(X^{\prime}, X\right) \mathrm{d} X^{\prime}=1
$$

Damping is included in the normal way during the passage from $E$ to $E^{\prime \prime}$. This leads to

$$
\Xi\left(X^{\prime}, X\right)=\frac{1}{2 A_{b^{\prime}} k_{b^{\prime}}} e^{-\mu D(\Phi(X), X)} \mathbb{1}_{b^{\prime \prime}}(\Phi(X)),
$$


where $\Phi$ is the ray tracing map from $E$ to $E^{\prime \prime}$. Inserting into (D.1), one obtains

$$
\varrho^{\prime}\left(X^{\prime}\right)=\{T \varrho\}\left(X^{\prime}\right)=\frac{1}{2 A_{b^{\prime}} k_{b^{\prime}}} \int e^{-\mu D(\Phi(X), X)} \mathbb{1}_{b^{\prime \prime}}(\Phi(X)) \varrho(X) \mathrm{d} X .
$$

In the orthogonal basis $F_{n}$, this reads

$$
T_{n^{\prime}, n}=\frac{\left(F_{n^{\prime}}^{\prime}, F_{n^{\prime}}^{\prime}\right)^{-1}}{2 A_{b^{\prime}} k_{b^{\prime}}}\left[\int F_{n^{\prime}}^{\prime}\left(X^{\prime}\right) \mathrm{d} X^{\prime}\right]\left[\int e^{-\mu D(\Phi(X), X)} \mathbb{1}_{b^{\prime \prime}}(\Phi(X)) F_{n}(X) \mathrm{d} X\right]
$$

and we obtain in our basis

$$
\int F_{n^{\prime}}^{\prime}\left(X^{\prime}\right) \mathrm{d} X^{\prime}=\frac{1}{\sqrt{A_{b^{\prime}} k_{b^{\prime}}}} \iint_{-k_{b^{\prime}}}^{k_{b^{\prime}}} P_{\beta^{\prime}}\left(\frac{p^{\prime}}{k_{b^{\prime}}}\right) \mathrm{d} p^{\prime} \mathrm{d} s^{\prime}=2 \sqrt{A_{b^{\prime}} k_{b^{\prime}}} \delta_{\beta^{\prime}, 0}
$$

and

$$
\begin{aligned}
\int e^{-\mu D(\Phi(X), X)} \mathbb{1}_{b^{\prime \prime}}(\Phi(X)) F_{n}(X) \mathrm{d} X \\
\quad=\frac{1}{\sqrt{A_{b} k_{b}}} \iint_{-k_{b}}^{k_{b}} e^{-\mu D(\Phi(X), X)} \mathbb{1}_{b^{\prime \prime}}(\Phi(X)) P_{\beta}\left(\frac{p}{k_{b}}\right) \mathrm{d} p \mathrm{~d} s \\
=\sqrt{\frac{k_{b}}{A_{b}}} \int_{-\pi / 2}^{+\pi / 2} h(\mu, \alpha) P_{\beta}(\sin \alpha) \cos \alpha \mathrm{d} \alpha .
\end{aligned}
$$

Together this gives

$$
T_{\left(b^{\prime}, \beta^{\prime}\right),(b, \beta)}=\frac{\delta_{\beta^{\prime}, 0}}{2 \sqrt{A_{b} A_{b^{\prime}}}} \sqrt{\frac{k_{b}}{k_{b^{\prime}}}}\left[\int_{-\pi / 2}^{+\pi / 2} h(\mu, \alpha) P_{\beta}(\sin \alpha) \cos \alpha \mathrm{d} \alpha\right] .
$$

Usually we do not have a single target edge $E^{\prime}$ as depicted in the left part of Fig. D.16, but instead the energy is spread out evenly over multiple edges in a target set $\mathcal{T}$ as sketched in the right part of Fig. D.16. If there is more than one target $E^{\prime}$, for each edge $E^{\prime}$ in the target set $\mathcal{T}$ we create an entry in the matrix representing the transfer operator $T$. We modify the matrix entry $T_{\left(b^{\prime}, \beta^{\prime}\right),(b, \beta)}$ by inserting an additional weight factor based on the phase space volume of the edges in the target set $\mathcal{T}$. The resulting matrix element $\tilde{T}_{\left(b^{\prime}, \beta^{\prime}\right),(b, \beta)}$ reads

$$
\tilde{T}_{\left(b^{\prime}, \beta^{\prime}\right),(b, \beta)}=\eta_{b^{\prime}} \frac{A_{b^{\prime}} k_{b^{\prime}}}{\sum_{\tilde{b} \in \mathcal{T}} A_{\tilde{b}} k_{\tilde{b}}} T_{\left(b^{\prime}, \beta^{\prime}\right),(b, \beta)} .
$$


Here, the transmission factor $\eta_{b^{\prime}}$ is an additional factor accounting for damping due to losses. This factor has to be determined from experience or experiment as explained in Sect. 4.2. The weighting factor in front of the $T$ matrix entry is based on the relative phase space volume of edge $b^{\prime}$ and reflects the idea that the RBE patch element acts like a chaotic scatterer that traps energy for some time. After a certain delay time, the trapped energy can escape to the adjacent regions with a rate based on the available phase space volume.

As an example, in the right part of Fig. D.16 we use the target set $\mathcal{T}=\{1,2,3, \ldots, 22\}$ and introduce the following 22 RBE patch elements: base $\{1\}$ and target $\mathcal{T}$, base $\{2\}$ and target $\mathcal{T}, \ldots$, base $\{22\}$ and target $\mathcal{T}$. Of special note is that we always include the base in the target set. We can then model that part of the energy is reflected. This kind of connectivity is also important for reciprocity: for zero damping and a basis consisting only of zero order Legendre polynomials, we expect that by appropriate weighting of the basis functions we can make both the column sums and the row sums (not with the same weighting) of the matrix $T$ to be equal to one.

We can also use more complicated schemes to transfer energies between different interface sections on the mesh. Let $\left\{J_{k}\right\}_{k=1}^{n}$ be the edge-index sets defining the $n$ interfaces. Let $S_{k^{\prime}, k}$ be the transition matrix whose entries are non-negative and whose column-sum is less than or equal to one. Then the matrix entries for the transfer operator are given by

$$
\tilde{T}_{\left(b^{\prime}, \beta^{\prime}\right),(b, \beta)}=\left[\sum_{\tilde{k}} S_{\tilde{k}, k}\right] \frac{S_{k^{\prime}, k} A_{b^{\prime}} k_{b^{\prime}}}{\sum_{\tilde{k}} S_{\tilde{k}, k} \sum_{\tilde{b} \in J_{\tilde{k}}} A_{\tilde{b}} k_{\tilde{b}}} T_{\left(b^{\prime}, \beta^{\prime}\right),(b, \beta)},
$$

where $b^{\prime \prime} \in J_{k}$ and $b^{\prime} \in J_{k^{\prime}}$. This formulation allows for energy transfer between multiple interfaces and for finer control over how much energy flows between the interfaces. Damping is taken into account by allowing the column sums of the matrix $S_{k^{\prime}, k}$ to be less than one. Although this more complicated formulation is not used in the calculations presented here, it is worthwhile to mention it as a possible direction for future investigation. 